\title{
Plasma Induced Hydrophilic Cellulose Wound Dressing
}

\author{
Zdenka Persin, Miran Mozetic, Alenka Vesel, Tina Maver, \\ Uros Maver and Karin Stana Kleinschek
}

Additional information is available at the end of the chapter

http://dx.doi.org/10.5772/55867

\section{Introduction}

The wound care industry is a highly diverse and competitive arena - including standard products such as dry bandages to sophisticated hydrogels and alginate dressings, and encompassing artificial skin and anti-infective used in wound care. A broad range of available modern dressings composed of layers possessing specific functionality has been placed on the market. Waddings and gauzes were replaced by new natural, synthetic and semi-synthetic biomaterials, which depending on their physical, chemical and technological properties are capable to absorb the exudate excess by maintaining the proper moisture environment on/in the wound. The use of textiles in medicine has a long tradition; specially woven textiles are mostly used.

Cellulose and its derivatives are very often used as a functional part of different wound dressing material e.g. as hydrogels, fibre, non-woven. Cellulose micro-fibrillar structure control the accessibility of the surface, while the bulk polar groups (-OH groups) ensure an attraction of the water molecule to the cellulose fibre (hydrogen bonds). Generally different standard chemical pre-treatments (i.e. alkaline washing, bleaching, and slack-mercerization) are used to improve the sorption capacity [1-4], but besides the ecological pollution, they also worsen fibres mechanical properties to a large extent. On the subject of the latter, plasma treatments are known to be rather harmless surface activation procedures leading to enhancement of hydrophilic properties; the best results are obtained using highly nonequilibrium oxygen plasma. Reactive particles created in plasma readily interact with cellulose resulting in formation of surface functional groups, as well as etching. The properties of plasma treated materials depend on the processing parameters and are measured using surface sensitive techniques. Numerous authors worldwide addressed modification of cellulose and other suitable materials by gaseous plasma treatment [5-16]. 
Unfortunately, however, only few authors are dared to measure plasma parameters so the results obtained by different authors cannot be directly compared. Many authors addressed surface properties such as the surface free energy [17-18], and surface functional groups. The surface free energy is usually determined from measurements of the contact angle of a suitable liquid or by more sophisticated techniques [19-20], while the appearance of specific functional groups has been determined by X-ray Photoelectron Spectroscopy (XPS) [6,21-23], Secondary Ion Mass Spectroscopy (SIMS) [24] and Furrier Transform Infrared Spectroscopy (FTIR) [21,25], just to mention the most commonly used ones. The modification of surface morphology of plasma treated textiles for wound dressings has been often studied by Scanning Electron Microscopy (SEM) [23-25] and Atomic Force Microscopy (AFM) [22, 2627].

A variety of gases has been used for modification of surface properties in order to achieve better hydrophilicity. Many authors just use noble gases [9,21,28-29], but more oxidizing gases such as oxygen, air, carbon dioxide and water vapour often give better results, especially when the process speed is the merit. Traditionally, gaseous plasma was always created at low pressure in vacuum compatible chambers. In the past couple of decades, however, plasmas which run at atmospheric pressure are becoming more and more popular. In fact, many resent works on modification of organic materials by gaseous plasma treatment report application of a kind of atmospheric gaseous plasma. As mentioned earlier, the richness of available plasmas as well as the lack of experimental techniques used by different authors prevents both direct comparison of reported result as well as good insight in the phenomena taking place during plasma treatment of textiles and similar materials. An important scope of this chapter is to explain some basic considerations about plasma textile interaction and give hints for optimal utilization of this advanced technique for modification of materials.

\section{Plasma treatment of cellulose wound dressing}

The hydrophilicity of organic materials is improved dramatically by incorporation of oxygen rich functional groups on to the surface, so a natural choice of gas used in plasma treatment of materials is oxygen. In thermodynamic equilibrium and at room temperature oxygen gas contains only neutral oxygen molecules. According to the basic law of statistical mechanics the molecules move randomly, collide with each other and the basic kinetic properties are described well taking into account the approximation of an ideal gas. The distribution of molecules over the kinetic energy is given by Maxwell-Boltzmann law [30] and the average random velocity is calculated as:

$$
v=\sqrt{8 k T /(\pi m)}
$$

The random velocity only depends on the gas temperature. At room temperature the average random velocity of oxygen molecules is about $440 \mathrm{~m} / \mathrm{s}$, while at $1000 \mathrm{~K}$ it is about $810 \mathrm{~m} / \mathrm{s}$. Oxygen molecules reach the surface of any material with different velocities taking into account the Maxwell-Boltzmann distribution, but as long as the gas temperature is close 
to the room temperature the velocity is too low to bring enough energy for chemical reactions on the surface of cellulose materials. Increasing gas temperature or the temperature of the cellulose material the available energy can become large enough to overcome the potential barrier and chemical interaction between oxygen molecules and cellulose is observed. The chemical interaction is highly exothermic so the material is locally heated due to a chemical reaction, which results in even higher chemical affinity resulting in more exothermic reactions etc. so finally the control of oxidation mechanism is lost and the chemical reactions leading to the oxidation of cellulose become very intensive. A common expression for such experimental conditions is burning. Treatment of textiles by oxygen gas at equilibrium conditions is therefore not useful because the oxidation is difficult if not impossible to control. One could use oxygen at very low concentration in order to avoid too extensive chemical reactions, but the results are usually not optimal. This is the reason why equilibrium gaseous treatment has not been used for introduction of highly polar oxygenrich functional groups onto the surface of organic materials. Instead, non-equilibrium gases should be used.

A typical example of non-equilibrium gas is gaseous plasma. It can be created by different means but the most common method is application of a gaseous discharge. In a gaseous discharge an electric field is applied and the gaseous molecules ionize to positive ions and free electrons. Charged particles are accelerated in electric fields and collide with neutral gaseous molecules as well as surfaces. If the kinetic energy of charged particles is above the ionization threshold the collisions will result in ionization of a neutral molecule and thus multiplication of charged particles. Particles with opposite charges attract each other and may or may not neutralize upon collisions. The neutralization may occur either in the gas phase or on surfaces. After turning on the electric field the multiplication of charged particles is quickly balanced by the loss due to neutralization so rather steady concentration of charged particles is established. The concentration depends on numerous parameters and the discharge power is only one of them.

Ionization is definitely the most important collision event since it allows for sustaining the discharge and thus transformation of oxygen gas into the state of plasma. In practical applications, however, it has a minor role in modification of organic materials. Other collisions are often much more important in order to make oxygen a suitable medium for surface functionalization of organic materials. Such collisions include excitation and dissociation of oxygen molecules as well as excitation of oxygen atoms. Namely, oxygen molecules are rich in excited states and so are oxygen atoms. Many excited states are metastable which means that do not decay in a short time by electrical dipole radiation. Such metastable molecules or atoms are not only more reactive than the counterparts in the ground state, but also play a very important role in formation of oxygen particles in highly excited states. Table 1 presents some most important excited states of oxygen molecules, while appropriate values for oxygen atoms are summarized in Table 2.

Examination of Table 1 reveals important data which should be taken into account when dealing with application of oxygen plasma for modification of cellulose materials. The 
ground state has the potential energy $0 \mathrm{eV}$ by definition. A variety of vibrational and rotational excited states are not shown in Table 1 due to the clearness. Also, the population of oxygen molecules in highly excited vibrational states is usually negligible in plasma suitable for modification of organic materials due to super elastic collisions between vibrational excited molecules and atoms which are very likely to appear in oxygen plasma [31]. At such super-elastic collisions a part of molecular vibrational energy is transferred to the kinetic energy of colliding particles. Since the cross-section for super-elastic collisions between vibrational excited oxygen molecules and neutral oxygen atoms is very large, this event effectively reduces the number of oxygen molecules in highly vibrational excited states so any interaction between such molecules and organic materials is regarded not important. The rotational states are usually coupled rather well with translational ones so the rotational population is similar to that calculated using equations of equilibrium thermodynamics. In fact, the rotational population detected by high resolution optical emission spectroscopy is often used for estimation of the neutral gas kinetic temperature in gaseous plasmas.

\begin{tabular}{lcc}
\hline Excited state & Optical symbol & Excitation energy \\
\hline Ground & $\mathrm{X}^{3} \Sigma_{\mathrm{g}}{ }^{-}$ & $0 \mathrm{eV}$ \\
First excited & $\mathrm{a}^{1} \Delta_{\mathrm{g}}$ & $1 \mathrm{eV}$ \\
Second excited & $\mathrm{b}^{1} \Sigma_{\mathrm{g}}{ }^{+}$ & $2 \mathrm{eV}$ \\
& $\mathrm{B}^{3} \Sigma_{\mathrm{u}}{ }^{-}$ & $6 \mathrm{eV}$ \\
Ground ionized & $\mathrm{X}^{2} \Pi_{\mathrm{g}}$ & $12 \mathrm{eV}$ \\
First ionized & $\mathrm{a}^{4} \Pi_{\mathrm{u}}$ & $16 \mathrm{eV}$ \\
Second ionized & $\mathrm{A}^{2} \Pi_{\mathrm{u}}$ & $17 \mathrm{eV}$ \\
Dissociation energy & & $4.5 \mathrm{eV}$ \\
\hline
\end{tabular}

Table 1. Most important excited states of oxygen molecules and their excitation energies

\begin{tabular}{lcc}
\hline Excited state & Optical symbol & Excitation energy \\
\hline Ground & $2 \mathrm{~s}^{2} 2 \mathrm{p}^{4} \mathrm{P}_{2}$ & $0 \mathrm{eV}$ \\
First excited & $2 \mathrm{~s}^{2} 2 \mathrm{p}^{4} \mathrm{D}$ & $2 \mathrm{eV}$ \\
Second excited & $2 \mathrm{~s}^{2} 2 \mathrm{p}^{4} \mathrm{~S}$ & $4 \mathrm{eV}$ \\
Metastable highly excited & $2 s^{2} 2 p^{3}\left({ }^{4} \mathrm{~S}^{\circ}\right) 3 s^{5} \mathrm{~S}^{0}$ & $9.1 \mathrm{eV}$ \\
& $2 s^{2} 2 p^{3}\left({ }^{4} \mathrm{~S}^{\circ}\right) 3 s^{3} \mathrm{~S}^{0}$ & $9.5 \mathrm{eV}$ \\
& $2 s^{2} 2 p^{3}\left({ }^{4} \mathrm{~S}^{\circ}\right) 5 s^{5} S^{0}$ & $12.7 \mathrm{eV}$ \\
\hline
\end{tabular}

Table 2. Most important excited states of oxygen atoms and their excitation energies

More important that vibrational states are electronically excited states. Oxygen molecules are famous of two metastable states which appear at the excitation energies of about 1 and 2 $\mathrm{eV}$, respectively (Table 1). Especially the first excited state has a very long radiative life-time of nearly one hour. The life-time of second excited state is much shorter at about ten seconds, but still very long for the atomic world. The molecules in excited states are definitely chemically more reactive than those found in the ground state, but very little 
work has been done on explanation of interaction mechanisms between electronically excited oxygen molecules and organic materials. Many authors neglect such an interaction since they claim other particles found in oxygen plasma are more suitable for modification of surface properties. All authors, however, agree that metastable oxygen molecules play an important role in dissociation events. Table 1 reveals that the dissociation energy of oxygen molecules is about $4.5 \mathrm{eV}$. An electron colliding with the molecule in the ground state will thus need kinetic energy higher than this value to be capable of a dissociation collision. The dissociation energy of excited molecule, however, is much lower than for a molecule in the ground state: for a molecule in the first excited state the dissociation energy is about $3.5 \mathrm{eV}$, and for a molecule in the second excited state it is about $2.5 \mathrm{eV}$. The difference in excitation energies of molecules found in the ground or excited states does not seem extremely important, but in fact it is due to two important considerations: (i) the high-energy tail of the electron energy distribution function in gaseous plasma is exponential, and (ii) the dissociation cross-section just above the threshold increases extremely steep with increasing kinetic energy of an electron. These two considerations indicate that the dissociation of molecules in oxygen plasma will be a very probable event due to step-like excitation. The result of the oxygen molecule dissociation is formation of neutral oxygen atoms in the ground state. Taking into account the upper considerations the production of atoms will be extensive in oxygen plasma created by any suitable electrical discharge. In spite of this fact the density of neutral oxygen atoms in technological plasmas vary enormously between different configurations. Neutral oxygen atoms tend to recombine (associate to form a molecule). The recombination event may take place either in the gas phase or on surfaces. The recombination event is simply described by reaction $\mathrm{O}+\mathrm{O} \rightarrow \mathrm{O}_{2}$. Such a simple denotation is, however, wrong since it does not take into account the conservation of energy and momentum. According to Table 1 a large amount of energy is released at a recombination event. If the recombination results in formation of a molecule in the ground state the amount of energy released is $4.5 \mathrm{eV}$. If a molecule in an excited state is formed the appropriate amount of released energy is accordingly lower. In any case, the released energy cannot be transferred into the kinetic energy of newly born oxygen molecule due to the conservation of momentum. This is why simple recombination as mentioned above does not occur in oxygen at low pressure. A possible recombination mechanism which can occur in the gas phase is radiative recombination $\mathrm{O}+\mathrm{O} \rightarrow \mathrm{O}_{2}+\gamma$, where $\gamma$ is a newly born light quantum (a photon) which takes the excessive energy. In this case two particles are formed at the recombination event so there are no problems with conservation of the momentum. The probability of such reaction depends on the quantum characteristics of particles involved. For the case of recombination of neutral oxygen atoms such reactions are highly improbable so neutral oxygen atoms are regarded stable in vacuum.

Oxygen atoms may recombine in the gas phase at so called three-body collisions $\mathrm{O}+\mathrm{O}+\mathrm{O}_{2}$ $\rightarrow \mathrm{O}_{2}+\mathrm{O}_{2}$. In such a case, the released energy is shared between the two molecules. The molecules may gain the released energy either as kinetic or excitation energy. The sharing of released energy again depends on the quantum characteristics of particles involved, and very typically the newly born molecule is found in an excited state. Such a reaction is actually an important source of excited molecules in plasma afterglows [32]. Since a three 
body collision is required the loss of atoms through this channel depends on the pressure (or the density of gaseous molecules). As long as the pressure is below several mbar the probability of three-body collisions is very low and this channel is regarded a marginal one at low-pressure plasmas. The opposite is true at atmospheric pressure where the probability for such collision is very high and practically any collision regardless the kinetic energy and momentum is highly probable. This is an important reason for observed large gradients on reactive gaseous particles in atmospheric plasmas.

Low-pressure plasma is therefore characterized by an absence of atomic loss mechanisms in the gas phase. The only channel for atom recombination in such plasmas is heterogeneous surface recombination. The reaction is written as $\mathrm{O}+\mathrm{O}_{\text {(surf) }} \rightarrow \mathrm{O}_{2 \text { (surf). In the case of surface }}$ recombination the excessive energy released is shared between the atoms in the solid material and the newly born molecule. An important consideration about surface recombination is the presence of oxygen atoms on the solid material surface. Namely, the recombination is unlikely to occur if atoms are not stuck on the surface. The recombination mechanisms have been explained neglecting details many decades ago. A couple of recombination mechanisms have been proposed: (i) Langmuir-Hinshelwood and (ii) EleyRideal mechanisms. The first one predicts adsorption of at least two atoms on the solid state surface, migration to an appropriate site and association of two atoms into a molecule. Since both atoms involved are already thermalized on the surface the newly born molecule leaves the surface in the ground state. In the case of Eley-Rideal mechanism oxygen atom is adsorbed on the surface where it waits for an atom from the gas phase and recombines to a molecule immediately (before the atom from the gas phase manages to thermalize on the solid surface). In this case the newly born molecule is likely to leave the surface in an excited state.

Whatever the recombination mechanism is, the reaction probability and thus the loss of atoms on surfaces depends on the ability of adsorption and the life-time of an adsorbed atom. Many materials tend to chemisorb atoms. Chemisorption stands for formation of a chemical bond between an oxygen atom and atoms on the surface. A good example is interaction between a metal and an oxygen atom. The oxygen atom is chemically bonded to the metal surface where it waits for other atoms to interact with them and form a molecule. The recombination probability on such surfaces is therefore very large. Appropriate literature reports experimentally determined values which are of the order of 0.1 for many metals. The consequence of such extensive surface recombination is heating of metals in atom rich plasma. This effect is used by catalytic probes - the thermal load onto a selected catalyst is a measure of the $\mathrm{O}$ atom density in the vicinity of the probe [33-36]. The exception to highly recombinant metals is a group of metals which form extremely stable oxides such as aluminium, titanium and alike. In such cases the atoms form very stable compounds and are thus not any more available for recombination. Recombination coefficients for such materials are orders of magnitude lower that for metals mentioned above.

Materials which do not chemisorb oxygen atoms are famous of having low coefficients for heterogeneous surface recombination of oxygen atoms. Apart from oxide ceramics the group of materials inert to chemisorption of oxygen atoms includes glasses. A glass is often a mixture 
of metal oxides which are chemically extremely inert and thus unlikely to attract neutral oxygen atoms. Such inertness is preserved also to elevated temperatures so different glasses are often used as building materials for plasma reactors where high density of neutral oxygen atoms is required. The recombination coefficient for neutral oxygen atoms on glasses depends on the type of material as well as surface roughness and is often as low as $10^{-4}$. A typical lowpressure plasma reactor made from glass is shown in Figure 1. In such glass chambers it is common to achieve very high dissociation fractions of oxygen molecules even at moderate discharge power. Values exceeding $10 \%$ have been reported by different authors [37-39]. The reasons are obvious from upper considerations: a very low recombination coefficient on plasma facing materials, negligible loss in the gas phase and favourite production by dissociation of molecules taking into account their metastable excited states.

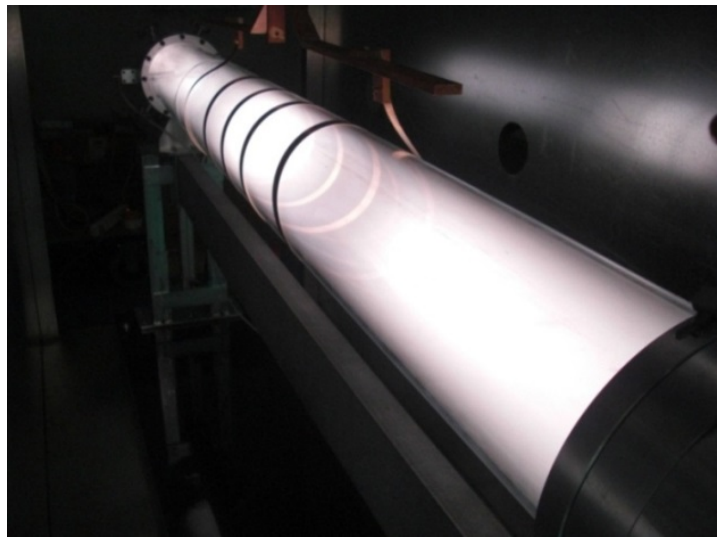

Figure 1. A photo of plasma created in a glass discharge chamber

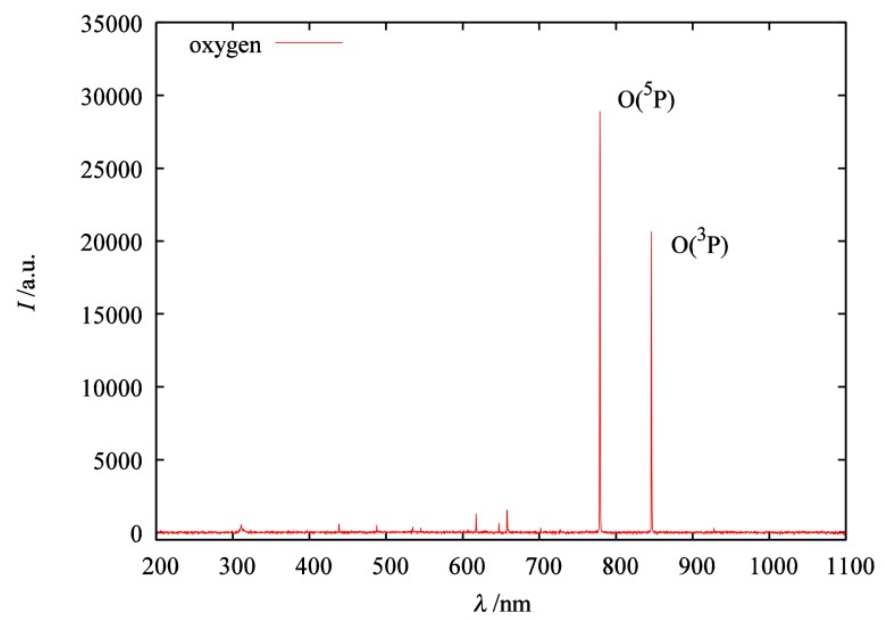

Figure 2. An optical spectrum acquired in oxygen plasma created by an electrode-less discharge in a glass chamber 
Not only oxygen molecules, but also neutral atoms have a lot of excited states. Table 2 represents the most important ones. The first excited state is at the excitation energy of about $2 \mathrm{eV}$ and is metastable with the characteristic radiation life-time of about 1 second. As mentioned earlier this is a very long life for atomic world. Excited states which are not metastable de-excite by electrical dipole radiation in a fraction of a microsecond. The second excited state is also metastable, and the radiation life-time is about $100 \mathrm{~s}$. Many other excited states are not metastable which in practice means that an atom found in such an excited state de-excites quickly by emission of a light quantum. An important metastable excited state is found at very high excitation energy of about $9 \mathrm{eV}$. The existence of such a state allows for excitation of oxygen atoms from this metastable state to very high levels and even ionization. The ionization energy of an oxygen atom is about $13.6 \mathrm{eV}$. Taking into account that one has to dissociate molecule first and then ionize the atom in order to get a positively charged oxygen atomic ion it is rather improbable that such ions would abound in plasma created by an electrical discharge of a low or moderate power. Still, the existence of metastable states definitely makes ionization of oxygen atoms easier. In practical applications it does not really matter whether the positive ions are atomic or molecular, but the distribution of atoms over excited states is important since the chemical reactivity of atoms depends largely on the excitation energy. Due to the high energies involved one would think that it is unlikely to excite atoms above the level of the $2 s^{2} 2 p^{3}\left({ }^{4} S^{\circ}\right) 3 s^{5} S^{0}$ using low power discharges, but such an assumption is not justified experimentally. Experiments with a simple optical emission spectrometer clearly show abundance of atoms in highly excited states. Figure 2 represents an optical spectrum of plasma created in oxygen by highfrequency discharge in a glass discharge chamber. In this spectrum atomic oxygen lines arising from transitions of highly excited atoms prevail indicating the presence of such atoms. Since the excitation energy of radiative excited states exceeds $11 \mathrm{eV}$ it is clear that excitation is a step-like process: after successful dissociation of a molecule an atom is excited to a metastable state of low energy, such a state is excited to a metastable state of high energy and finally an atom is excited to a radiative state. After emission of a photon the atom does not de-excite to the ground state, but rather to the metastable state so another excitation to a radiative state is likely to occur. The spectra such as one presented in Figure 2 therefore represent a good fingerprint of the existence of highly excited metastable atoms in oxygen plasma.

Plasma is usually defined as an ionized gas. Taking into account upper considerations, such a simple definition is hardly useful in practical applications such as activation of polymer materials. Namely, the concentration of neutral reactive gaseous particles is often orders of magnitude larger than the concentration of charged particles. From this point of view it may be more convenient to define plasma as a state of gas which is essentially close to room temperature, but the chemical reactivity is as high as the parent gas at thousands of degrees if not higher. A huge difference between the gas temperature and chemical reactivity steams from the fact that, unlike the case of equilibrium gases, high temperature is not a necessary condition for achieving an appropriate concentration of highly reactive gaseous particles. Instead of high temperatures, non-equilibrium gases take advantage of the fact that the electrons are found at extremely high temperatures while the rest of the gas practically 
remains at room temperature. The reason for a huge difference between the electron temperature which easily exceeds $10,000 \mathrm{~K}$ and the gas temperature comes from two facts: (i) electrons are easily accelerated to high energy in electrical fields, and (ii) the kinetic energy coupling between electrons and other particles is negligible. In a simple DC (directcurrent) discharge the electrons are born on the cathode by secondary electron emission due to bombardment of the cathode by positive ions. All charged particles accelerate in the potential fall near the cathode. Electrons are accelerated towards plasma while positively charged particles are directed towards the cathode and thus cannot contribute to gas heating. The energy positive ions gain by passing the cathode sheath is therefore used for emission of free electrons, but the major part is simply lost for heating of the cathode material. Electrons born by secondary emission are accelerated towards the plasma. They gain a lot of kinetic energy which they slowly loose at elastic collisions with other electrons from plasma. Since the energy transfer between electrons is very intensive, fast electrons born on the cathode quickly thermalize in plasma thus heating other electrons. The electrons gain Maxwell-Boltzamann distribution throughout the volume of gaseous plasma. Since they are continuously heated by fast electrons from the cathode their temperature remains very high despite of the loss of kinetic energy at inelastic collisions with gaseous molecules. This is why electrons are usually at very high temperature as compared to other particles present in non-equilibrium gaseous plasma. Similar observations are valid for capacitively coupled high-frequency discharges except that the electrons are accelerated in DC self-bias sheath next to the smaller (powered) electrode. The physics of such discharges has been studied to detail in [40-42]. The heating of electrons follows a different mechanism in inductively coupled discharges, especially those created in pure H-mode [43]. In such cases the electrons follow oscillations of the electrical field induced by oscillating magnetic field. Since the magnetic field oscillates in the entire volume of the discharge the electrons are accelerating in the entire volume, too. No acceleration across sheath is required so plasma can be created in an absence of any electrode. This is a favourite property since electrodes often represent a major sink for neutral oxygen atoms. As mentioned above the recombination of neutral oxygen atoms on metallic surfaces is always orders of magnitude more important than on glass surfaces. Another advantage of inductively coupled discharge is an absence of ion acceleration. If the frequency of the oscillating field is large enough (typically above $1 \mathrm{MHz}$ ) the ions cannot follow oscillations of the local electric field since the frequency is well above the resonant one. Massive ions therefore gain a negligible energy from a high-frequency electric field so they remain at room temperature. On the other hand, electrons are light enough to be able to follow the oscillations. The kinetic energy they gain from the electric filed is spent for their multiplication at ionizing collisions as well as for excitation and dissociation of oxygen molecules. Inductively coupled plasmas are therefore very suitable sources of highly excited oxygen atoms which are chemically extremely reactive.

In the first part of this chapter we clearly stressed an importance of avoiding high temperature during oxidation of materials. Although gaseous plasma is basically at room (or little elevated) temperature the heating of materials exposed to gaseous plasma is not always negligible. We already mentioned heating of materials with a high coefficient for 
heterogeneous surface recombination. A variety of surface physical and chemical reactions are exothermic. Among physical reactions one should stress weak bombardment of a material by positively charged ions, neutralization of charged particles, relaxation of metastables and recombination of neutral oxygen atoms. The thermal load obviously depends on the flux of reactive particles on to the surface, which in turn depends on the density of different particles in the gas phase.

Since the electron temperature $\left(T_{e}\right)$ in plasma is much larger than the positive ion temperature and since they have a favourable random velocity the electrons escape from plasma towards the surfaces of the discharge vessel much faster than the positive ions. The surface of plasma facing components thus becomes negatively charged against gaseous plasma. The potential difference between plasma $\left(V_{p}\right)$ and the surface $\left(V_{f}\right)$ is created in such a way to repel most electrons except the fastest and accelerate ions. In steady state conditions the flux of electrons on to the surface is equal to the flux of positive ions. Obviously, the potential difference between unperturbed plasma and the solid surface increase with increasing electron temperature. In the first approximation the potential difference is calculated using equation:

$$
V_{p}-V_{f}=\frac{k T_{e}}{2 e_{o}} \ln \left(\frac{m_{+}}{2 m_{e}}\right),
$$

Positively charged ions are therefore accelerated towards the surface. They impinge the surface with kinetic energy which corresponds to the potential difference as illustrated by equation (2). Their kinetic energy when impinging the surface therefore does not depend on the ion temperature in unperturbed plasma but rather on the potential difference between plasma and floating potentials. A typical value for the kinetic energy of ions impinging the surface is about $10 \mathrm{eV}$ taking into account the collision-less sheath approximation. This energy is not large enough to cause implantation of ions into a solid material, but causes heating of the solid material. Furthermore, positively charged ions are very likely to neutralize on solid surfaces. As mentioned before the surfaces are negatively charged against plasma so electrons abound on the surface. The observed probabilities for neutralization of slow ions on solid materials are actually very close to 1 . This means that an average ion reaching the surface of a plasma facing component brings energy equal to the sum of ionization and kinetic energies. Table 1 reveals that the ionization potential of oxygen molecule is about $12 \mathrm{eV}$ so the contribution of both energies is practically the same. In any case, interaction of positively charged ions with solid materials causes heating so highly ionized plasmas are not very suitable for treatment of delicate materials such as cellulose for wound dressings.

Not only charge particles but many other particles created in oxygen plasma are suitable for heating of cellulose materials. Highly excited atoms de-excite on the surfaces of practically any material at very high probability so they directly contribute to the heating. Their role, however, is difficult to quantify since the density of atoms in highly excited states is difficult to measure [44]. Theoretical simulations indicate that the density of oxygen atom 
metastables depends largely on the excitation energy and not so much on the radiative lifetime [32]. This finding is explained by a very high probability for quenching of highly excited states. Quenching stands for de-excitation by mechanisms other than radiation. The concentration of excited oxygen atoms therefore depends on the electron density and the electron temperature. As long as the density of charged particles is rather low it is reasonable to assume a rather low density of atoms in excited states and thus negligible contribution to the heating of plasma facing materials.

Another source of heating of cellulose materials is heterogeneous surface recombination of neutral atoms in the ground state. As mentioned earlier the density of atoms in plasma created by electrode-less discharge in the glass tube is always high. The available energy dissipated at surface recombination is $4.5 \mathrm{eV}$. As explained above this energy is shared between the solid material and the newly born molecule. If the surface recombination was intensive, the heat load would have been high enough to cause immediate burning of cellulose materials. The experimental observations, however, show that cellulose materials do not burn in plasma containing large quantities of neutral oxygen atoms providing the density of charged particles is very low. This observation leads to the qualitative conclusion that the probability for heterogeneous recombination of neutral oxygen atoms in the ground state is rather low. The lack of data on reaction mechanisms is unfortunately severe. While recombination coefficients have been determined rather accurately on many other materials especially inorganic, a lot of work will have to be performed on recombination of neutral oxygen atoms in the ground state on organic materials in order to get a good insight into this mechanism and thus to estimate the corresponding thermal load. The same applies for excited oxygen molecules. In any case, these reactions are much less important for heating of cellulose materials than interaction of highly excited gaseous particles, so weakly ionized, highly dissociated oxygen plasma is suitable for modification of surface properties of cellulose materials.

The first effect of exposure of practically any organic material to oxygen plasma is functionalization of surface with oxygen rich functional groups. A usual technique for determination of functional groups is X-ray Photoelectron Spectroscopy (XPS) which is a very surface sensitive method with detection depth of only few nanometers. Therefore, it is a powerful technique for studying elemental composition and chemical state of the surfaces. In this chapter we present results obtained with our XPS device. The cellulose samples were analysed by TFA XPS instrument from Physical Electronics. The base pressure in the XPS analysis chamber was about $6 \times 10^{-10} \mathrm{mbar}$ and the samples were excited with $\mathrm{X}$-rays over a $400 \mu \mathrm{m}$ area using monochromatic $\mathrm{Al} \mathrm{K}_{\alpha 1,2}$ radiation at $1486.6 \mathrm{eV}$. The photoelectrons were detected by a hemispherical analyser, positioned at an angle of $45^{\circ}$ with respect to the surface normal. Survey-scan spectra were measured at a pass energy of $187.85 \mathrm{eV}$, while for $\mathrm{C} 1 \mathrm{~s}$ individual high-resolution spectra were taken at pass energy of $23.5 \mathrm{eV}$ and a $0.1-\mathrm{eV}$ energy step. An additional electron gun was used for surface neutralization of samples during the measurements. The measured spectra were analysed using MultiPak v7.3.1 software from Physical Electronics, which was supplied with the spectrometer. The highresolution carbon C 1s peaks were fitted with symmetrical Gauss-Lorentz functions. A 
Shirley-type background subtraction was used. Both the relative peak positions and the relative peak widths (FWHM) were fixed in the curve fitting process.

Cellulosic fibres, in a form of woven or non-woven textiles are one of the most used wound dressing base materials. Therefore, the cellulose material as viscose fibres in its non-woven form was studied, as produced by Kemex, the Netherlands. The surface mass was $175 \mathrm{~g} / \mathrm{m}^{2}$ (SIST ISO 3801), the thickness under normal conditions was $1.7 \mathrm{~mm}$ (SIST EN ISO 5084), and the air permeability $650 \mathrm{l} / \mathrm{m}^{2} \mathrm{~s}$ (DIN 53 887).

A typical survey spectrum of photoelectrons on cellulose material is presented in Figure 3. The spectrum is dominated by carbon and oxygen peaks. By far the largest carbon peak is marked as $\mathrm{C} 1 \mathrm{~s}$ and results from photoelectrons ejected from carbon 1s orbital. A much weaker peak is observed at the energy of about $25 \mathrm{eV}$. Several oxygen peaks are observed in the spectrum as well and the O1s predominates. The survey spectrum is suitable for estimation of the material composition in the surface layer with the thickness of few nanometres but tells nothing about the functional groups. In order to reveal the chemical structure one should acquire a high-resolution spectrum of $\mathrm{C} 1 \mathrm{~s}$. Such a spectrum is shown in Figure 4 for the case of a very flat cellulose material while a corresponding spectrum for cellulose material used for wound dressing is shown in Figure 5. The curve presented in Figure 4 reveals three different functional groups. The major peak at about $286.4 \mathrm{eV}$ corresponds to $\mathrm{C}-\mathrm{O}$ functional group which abounds in cellulose materials. A well distinguished peak at $284.8 \mathrm{eV}$ corresponds to the C-C bonds, while the third one which appears as a shoulder at about $287.9 \mathrm{eV}$ corresponds to the $\mathrm{O}-\mathrm{C}-\mathrm{O}$ bond. The spectrum presented in Figure 5, on the other hand, does not reveal well expressed peaks due to the artefacts typical for this sort of surface characterization. The major reason for unclear peaks is a rich roughness of the material. Detailed explanation of such deviations is presented elsewhere [45].

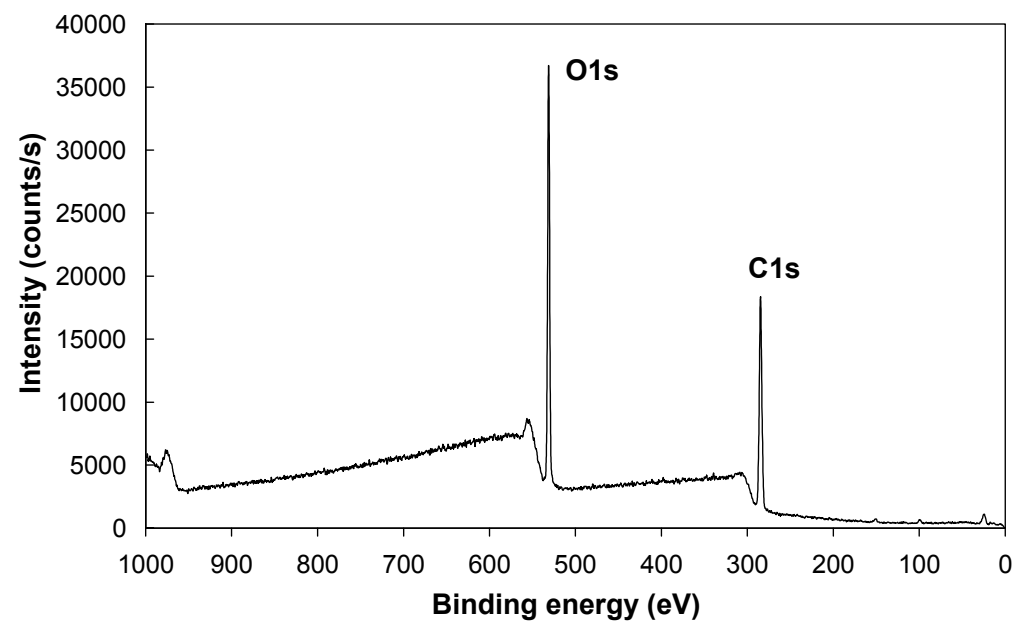

Figure 3. A survey spectrum of cellulose material 


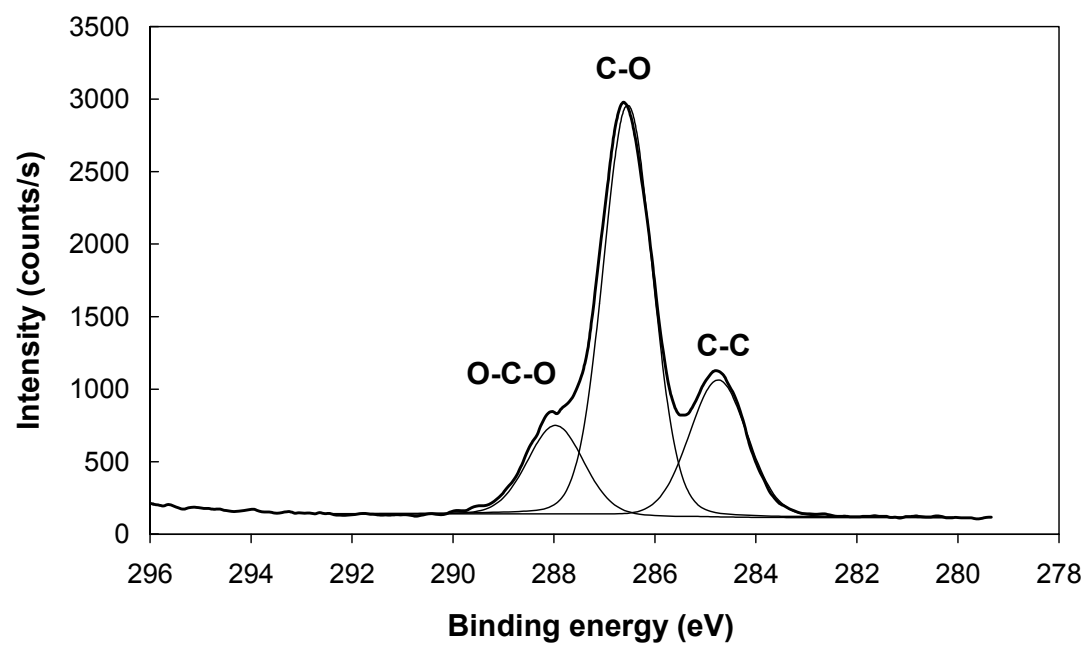

Figure 4. A high-resolution XPS spectrum obtained on a cellulose film

The survey spectrum of cellulose used for wound dressings treated by oxygen plasma is not much different from the one presented in Figure 3 so it is not shown in this chapter. Instead, a comparison of atomic composition between non-treated and oxygen plasma treated cellulose is presented in Table 3. Of particular importance is the ratio between oxygen and carbon atoms as detected by XPS and presented in Table 3. One could observe a large difference of almost $20 \%$. Here, it is worth stressing that the escape depth of photoelectrons is several $\mathrm{nm}$ so the experimental technique give somehow averaged value over the few $\mathrm{nm}$ thick surface layer. Taking into account gradients of oxygen concentration (it is the largest on the very surface) one could qualitatively argue that the actual oxygen concentration on the very surface is much larger than shown in Table 3. Any gradients in the elemental composition in a thin surface film could be estimated by tilting the sample during XPS characterization but such procedure is efficient only in the case of very flat samples. Cellulose materials used for wound dressings definitely do not fulfil this condition.

\begin{tabular}{lccc}
\hline \multirow{2}{*}{ Sample treatment } & \multicolumn{3}{c}{ Surface composition (at. \%) } \\
\cline { 2 - 4 } & $\mathrm{C}$ & $\mathrm{O}$ & $\mathrm{O} / \mathrm{C}$ ratio \\
\hline Non-treated & 57.2 & 42.2 & 0.73 \\
Oxygen plasma treated & 52.1 & 47.9 & 0.91 \\
\hline
\end{tabular}

Table 3. Surface composition of non-treated and oxygen plasma treated cellulose fabric

Figure 6 represents a typical high resolution $C$ 1s peak of cellulose material which has been treated by oxygen plasma. Unlike the non-treated material the peak now contains four different sub-peaks. The newly formed sub-peak at high binding energies in Figure 6 corresponds to a new functional group which is formed on the cellulose material during treatment with oxygen plasma and it corresponds to the carboxyl functional group 


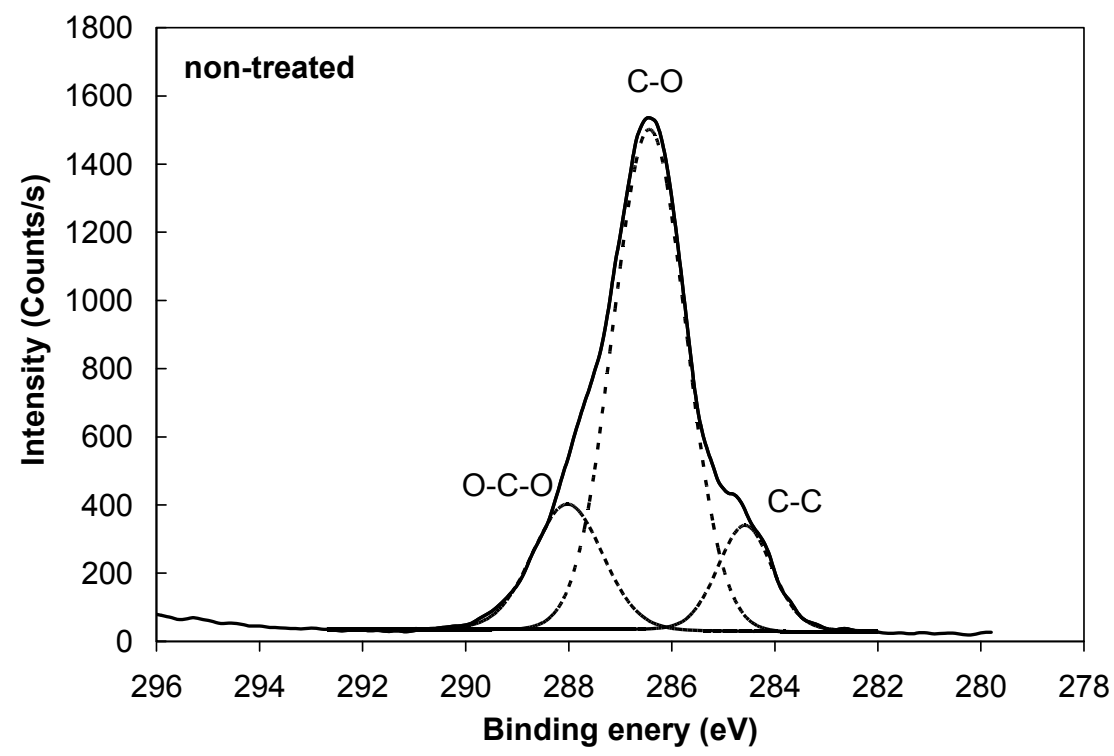

Figure 5. A high-resolution XPS spectrum obtained on cellulose material used for wound dressing

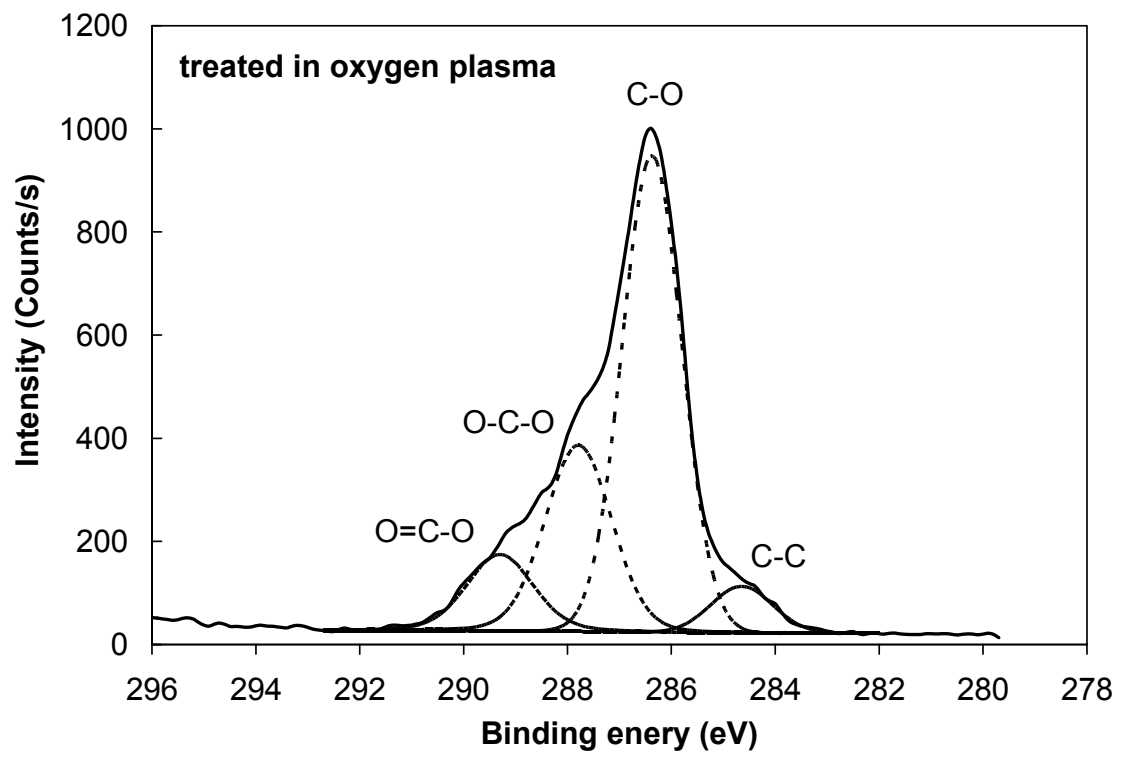

Figure 6. A high-resolution XPS spectrum obtained on oxygen plasma treated cellulose material used for wound dressing 
$\mathrm{O}=\mathrm{C}-\mathrm{OH}$. Furthermore, the sub-peak $\mathrm{C}-\mathrm{C}$ in Figures 5 and 6 is much smaller after plasma treatment, while the O-C-O sub-peak is enlarged somehow and it can contain also contribution of $\mathrm{C}=\mathrm{O}$ bonds. Taking into account upper considerations one can easily speculate that the $\mathrm{C}-\mathrm{C}$ bonds completely vanish from the very surface of cellulose material upon oxygen plasma treatment. Plasma treatment thus facilitates formation of a functional group which is extremely polar and thus increases the polar component of the surface energy which in turn leads to improved soaking properties of the cellulose material.

XPS results indicate information of surface functional groups which should influence sorption properties of cellulose materials. One of the best techniques for studying the sorption properties is contact angle measurements. This is actually one of the oldest experimental techniques, and probably the simplest. In its basic configuration it is just a drop of water placed onto a surface of the material for which the surface properties are to be determined. Large contact angles of a water drop indicate hydrophobic character of a material, while small contact angles are typical for hydrophilic materials. Such a simple technique is applicable for rapid estimation of the surface character but it is not accurate and usually fails at attempt to use it on porous materials such as cellulose for wound dressing. In order to overcome the limitations of the method in its original form more sophisticated versions have been developed and applied by different authors [46-51]. Although large progress has been obtained in the last decades the technique is still regarded semiquantitative due to complexity of interaction between liquid drops and solid materials. In order to give a comprehensive explanation of this technique we present different models currently used for quantification of measured results.

Contact angle measurements, which can be used to characterize the hydrophobic/hydrophilic properties of surfaces and from which changes in adhesion can be calculated [52], are frequently used to evaluate the effect of chemical changes on the adhesive properties of polymer surfaces. Various approaches for further analyses of these properties have appeared. It is generally agreed that the surface free energy contains the non-polar and polar components. In this sense, the dispersive (Lifshitz van der Waals, LW) and polar (acid-base, $\mathrm{AB}$ ) interactions are known. The most common mathematical models used for determining the surface free energy (SFE) are the ones suggested by Fowkes [5354], Zisman [55], Kwok and Neumann [56], denoted "Equation of state", Rabel [57], Kaelble [58-59], Owens and Wendt [60], denoted "OW", and van Oss, Chaudhury and Good [61-63], denoted "vOGC".

The determination of the solid SFE is a complex procedure, and it cannot be measured directly. As such, the evaluation technique consists of three stages i) characterisation of test liquids using Wilhelmy plate (platinum and PTFE) and Du-Nuoy ring methods, ii) contact angle determination by bringing various standard liquids in contact with the solid surface, and iii) evaluating the SFE by using an appropriate mathematical model. Prior to contact angle determination, standard liquids must be investigated in order to determine their surface tensions (SFT) and their dispersive and polar components. Test liquids with different surface tensions and different polar and dispersive components must be chosen in order to determine the SFE. 
The surface tensions of all liquids, except water, are determined using the Wilhelmy plate method according to DIN 53 914. The Du-Nuöy ring method according to DIN 53993 is applied for water. The pulling force of a liquid, when it is brought into contact with the lower edge of the platinum plate or the ring, is a linear measure of the liquid surface tension, $n$, i.e. for the plate:

$$
\gamma_{l}=\frac{F_{w}-F_{b}}{L \cdot \cos \theta}
$$

and for the ring

$$
\gamma_{l}=\frac{F_{\max }-F_{r}}{L \cdot \cos \theta}
$$

where $F_{w}$ is the force on the plate, $F_{\max }$ is the maximum pull on the ring, and $F_{b}$ and $F_{r}$ are the buoyancy corrections for the plate and the ring, respectively, $L$ is the wetted length $(\mathrm{mm})$ i.e. for the plate $L=2(a+b)$ where $a$ is the thickness and $b$ is the width of the plate, and for the ring $L=4 \pi R$ where $R$ is the major radius of the ring, and $\theta$ is the contact angle $\left({ }^{\circ}\right)$. Both the ring and the plate are made of platinum and it is assumed that the contact angle of all liquids on platinum is $0^{\circ}$.

The dispersive component of used test liquid is determined by measuring the contact angle between standard poly(tetrafluoroethylene) (PTFE) plate and liquid. According to Good et al. [64] PTFE is assumed to be capable only of dispersive interactions $\left(\gamma_{s}=\gamma_{s}{ }^{D}=18.0\right.$ $\mathrm{mJ} / \mathrm{m}^{2}$ and $\gamma s^{P}=0 \mathrm{~mJ} / \mathrm{m}^{2}$ ). The polar component of the liquid can be then calculated by the difference as:

$$
\gamma_{l}^{p}=\gamma_{l}-\gamma_{l}^{d}
$$

where $\gamma_{l}$ is the surface tension of the wetting liquid, $\gamma_{l}^{d}$ is the dispersive surface tension component of the wetting liquid, $\gamma_{l}^{p}$ is the polar surface tension component of the wetting liquid.

Contact angle between a cellulose sample and a liquid drop is determined using a capillary rise technique [65], applicable for porous materials. The samples weight is measured as a function of time during the adsorption of the liquid phase. The wetting rate curve $\left(\mathrm{m}^{2} / \mathrm{t}\right)$ is used to determine the contact angle using modified Washburn equation [66-67]:

$$
\cos \theta=\frac{m^{2}}{t} \cdot \frac{\eta}{\rho^{2} \cdot \gamma \cdot c}
$$

where $\theta$ is the contact angle between the solid and liquid phases, $m$ is the weight of the liquid that penetrates into sample, $t$ is the penetration time, $\eta$ is the liquid viscosity, $\rho$ is the liquid density, $\gamma$ is the surface tension of the liquid, and $c$ is a material constant. N-heptane or n-hexane (for which the contact angle on the solid sample is zero) is used as a liquid (for which the $\cos \theta=1$ ), in order to determine the material constant. 
Using water as the test liquid, hydrophilic/hydrophobic properties of solid surfaces are described by contact angle measurement results i.e. the hydrophilic $\left(\theta<90^{\circ}\right)$ or hydrophobic $\left(\theta>90^{\circ}\right)$. Contact angle measurement results, using various standard liquids, the samples surface energy can be determined using one among several common models for evaluating.

Fowkes SFE model [53-54] takes advantage of the theory that the molecular interactions contributing to surface tension are divided into two groups: dispersion (non-polar) interactions and polar interactions, i.e.:

$$
\gamma=\gamma^{d}+\gamma^{p}
$$

and that the adhesion $(W)$ between two materials 1 and 2 that interact only through dispersive forces $\left(\gamma_{p}=0\right)$ are estimated from:

$$
W_{12}=2 \sqrt{\gamma_{1}^{d} \gamma_{2}^{d}}
$$

The thermodynamic work of adhesion $W_{a}$ of a liquid with surface tension $n$ on a solid with surface tension $\gamma_{s}$ is defined by:

$$
W_{a}=\gamma_{s}+\gamma_{l}-\gamma_{s l}
$$

where $\gamma_{s l}$ is the solid-liquid interfacial tension.

The Young equation for the equilibrium contact angle on a solid surface is:

$$
\gamma_{l} \cos \theta=\gamma_{s, 0}-\gamma_{s l}-\pi_{s}
$$

where $\gamma_{s, 0}$ is the surface energy of the pure solid and $\pi_{s}$ is the surface pressure due to adsorption on the solid. On low-energy surfaces $\pi_{\mathrm{s}}$ is usually assumed to be insignificant, i.e. $\gamma_{s, 0}=\gamma_{s,}$. Combination with Equation (9) then yields the Young-Dupré equation:

$$
\mathrm{W}_{a}=\gamma_{l}(\cos \theta+1)
$$

The combination of Equations 8, 9 and 11 gives:

$$
\gamma_{s l}=\gamma_{s}+\gamma_{l}-2 \sqrt{\gamma_{s}^{d} \gamma_{l}^{d}}
$$

and

$$
W_{a}=\gamma_{l}(1+\cos \theta)=2 \sqrt{\gamma_{s}^{d} \gamma_{l}^{d}}
$$

or

$$
\gamma_{l}^{d}=\frac{\gamma_{l}^{2}(1+\cos \theta)}{4 \gamma_{d}^{s}}
$$


Therefore, when only dispersive interactions are significant, the dispersive component of the surface tension of the liquid can be determined by measuring the contact angle of a liquid on a solid with a known $\gamma_{s}^{d}$. The polar component of the surface tension is then determined by Equation (5).

The Owens-Wendt-Rabel-Kaelble (OW) SFE model [59-60] assumes that the polar interaction between solid and liquid can be estimated by a combination of $\gamma_{l}^{p}$ and $\gamma_{s}^{p}$. Thus, in the model it is proposed that the polar component can be described by a geometric mean (GM) approximation i.e.:

$$
\begin{gathered}
\gamma_{s l}=\gamma_{s}+\gamma_{l}-2 \sqrt{\gamma_{s}^{d} \gamma_{l}^{d}}-2 \sqrt{\gamma_{s}^{p} \gamma_{l}^{p}}, \\
W_{a}=\gamma_{l}(1+\cos \theta)=2 \sqrt{\gamma_{s}^{d} \gamma_{l}^{d}}+2 \sqrt{\gamma_{s}^{p} \gamma_{l}^{p}},
\end{gathered}
$$

where $\gamma_{s}^{p}$ and $\gamma_{l}^{p}$ are the polar components of the surface free energies of the solid and the liquid, respectively. Equation 15 can be used to determine $\gamma_{s}^{p}$ by measuring the contact angle of two liquids with known $\gamma_{l}^{d}$ and $\gamma_{l}^{p}$ (usually one with only dispersive and one with both dispersive and polar components). This approach has been criticized for being too simple because a polar, but purely acidic surface will show only dispersive interactions with acid solvents, and the same will be the case for the interaction of basic surfaces with basic solvents [68-69]. However, it has received widespread use as a method to characterise the adhesive properties of polymers, because at least two various liquids are necessary to measure the contact angle and then solve the system of equations. One of these liquids should be characterized by high value of $\gamma_{l}^{d}$ and low value of $\gamma_{l}^{p}$ and the second liquid inversely. The most often, water and diiodomethane were used as the pair of testing liquids.

In order to average the interactions with several solvents, Owens and Wendt [60] wrote Equation 16 in the linear form:

$$
y=a x+b,
$$

where

$$
\begin{gathered}
\mathrm{x}=\sqrt{\gamma_{s}-\gamma_{s}^{d}} \sqrt{\frac{\gamma_{l}^{p}}{\gamma_{l}^{d}}} ; \quad \mathrm{a}=\sqrt{\gamma_{s}^{p}}, \\
y=\frac{1+\cos \theta}{2} \frac{\gamma_{l}}{\sqrt{\gamma_{l}^{d}}}: \quad \mathrm{b}=\sqrt{\gamma_{s}^{d}} .
\end{gathered}
$$

Thus, in the OW approach, the contact angles of several liquids with known values of $\gamma_{l}, \gamma_{l}^{d}$ and $\gamma_{l}^{p}$ are measured after which data are fitted to straight lines (Equation 17). The slope and intercept of this line yields values of $\gamma_{l}^{d}$ and $\gamma_{l}^{p}$ (Equations 18 and 19).

Several professional devices are currently available for characterisation of surface properties by contact angle methods. The work described in this contribution has been performed 
using a professional device Kruss K12. The KRÜSS Tensiometer of the K12 series is the standard for the measurement of the surface and interfacial tension of liquids and solids. This instrument enables measuring surface and interfacial tension measurement of liquids, dynamic contact angle measurements, wetting behaviour of tablets, pharmaceutical active substances, auxiliaries, lacquers, paints, and printing inks, of fibre bundles, textiles, wetting and adhesion of coatings. The K12 Tensiometer has been specially designed for versatile and demanding applications in research of surface-active agent development, wetting properties of tablets, pharmaceutical active ingredients and auxiliary products, pigments, as well as in textiles, and monitoring surface optimization in the production of polymers and foils, lacquers, dyes, inks.

In order to evaluate the SFE of cellulose materials for wound dressing 6 different test liquids: water, ethylene glycol, ethanol, chloroform, tetrahydrofuran, and diiodomethane have been used. The results of surface tensions and contact angles of water and diiodomethane were used for geometric mean (GM) calculations (Equation 16). In OW calculations (Equation 18 and 19) the data for all six solvents were used.

The total surface tension and its dispersive and polar components have been measured and are found as follows: water $\left(\gamma=72 \mathrm{~mJ} / \mathrm{m}^{2} ; \gamma^{\mathrm{d}}=28 \mathrm{~mJ} / \mathrm{m}^{2} ; \gamma^{\mathrm{P}}=44 \mathrm{~mJ} / \mathrm{m}^{2}\right)$; Ethylene glycol $(\gamma=$ $\left.48 \mathrm{~mJ} / \mathrm{m}^{2} ; \gamma^{\mathrm{d}}=26 \mathrm{~mJ} / \mathrm{m}^{2} ; \gamma^{\mathrm{P}}=21 \mathrm{~mJ} / \mathrm{m}^{2}\right) ;$ Ethanol $\left(\gamma=22 \mathrm{~mJ} / \mathrm{m}^{2} ; \gamma^{\mathrm{d}}=20 \mathrm{~mJ} / \mathrm{m}^{2} ; \gamma^{\mathrm{P}}=2 \mathrm{~mJ} / \mathrm{m}^{2}\right)$; Chloroform $\left(\gamma=27 \mathrm{~mJ} / \mathrm{m}^{2} ; \gamma^{\mathrm{d}}=26 \mathrm{~mJ} / \mathrm{m}^{2} ; \gamma^{\mathrm{P}}=1 \mathrm{~mJ} / \mathrm{m}^{2}\right)$; Tetrahydrofuran $\left(\gamma=27 \mathrm{~mJ} / \mathrm{m}^{2} ; \gamma^{\mathrm{d}}=12\right.$ $\left.\mathrm{mJ} / \mathrm{m}^{2} ; \gamma^{\mathrm{p}}=15 \mathrm{~mJ} / \mathrm{m}^{2}\right)$, and Diiodomethane $\left(\gamma=51 \mathrm{~mJ} / \mathrm{m}^{2} ; \gamma^{\mathrm{d}}=51 \mathrm{~mJ} / \mathrm{m}^{2} ; \gamma^{\mathrm{p}}=0 \mathrm{~mJ} / \mathrm{m}^{2}\right)$. These measured values were used in evaluation of SFE using the mathematical model described above.

The cellulose samples were cut into rectangular pieces $\left(2 \times 5 \mathrm{~cm}^{2}\right)$ and hung up onto the sample holder in the Tensiometer Krüss K12 apparatus. Non-treated and plasma treated cellulose materials for wound dressing have been characterised by all six liquids. The wetting rise curves for water as the test liquid are presented in Figure 7. The results were statistically processed (a set of parallel measurements of wetting rise curves were performed until the standard deviation of calculated contact angle was less than $2^{\circ}$ ) and represent the average value of at least ten measurements of each sample.

The slope of curves presented in Figure 7 characterise the rate of water sorption. One can observe a significant difference in the rate of water sorption when comparing non-treated and plasma treated cellulose samples. In the case of plasma treated cellulose sample, the rate of water sorption was very fast at the beginning, while after a few seconds (e.g. $10 \mathrm{~s}$ ) the sorption slows and curve riches plateau indicating complete wetting of the material.

The wetting rise curves obtained using test liquids allow for determination of the contact angles which are used for evaluating the surface energies of non-treated and plasma treated materials, their dispersive and polar components. The contact angles are shown in Figure 8, while the corresponding values for surface energy, polar and dispersive components (as obtained by using Owens-Wendt-Rabel-Kaelble (OW) model) are summarised in Figure 9. As shown in Figure 8 the highest contact angles by all samples were determined using diiodomethane as a test liquid. The lowest values were obtained by ethylene glycol. The 


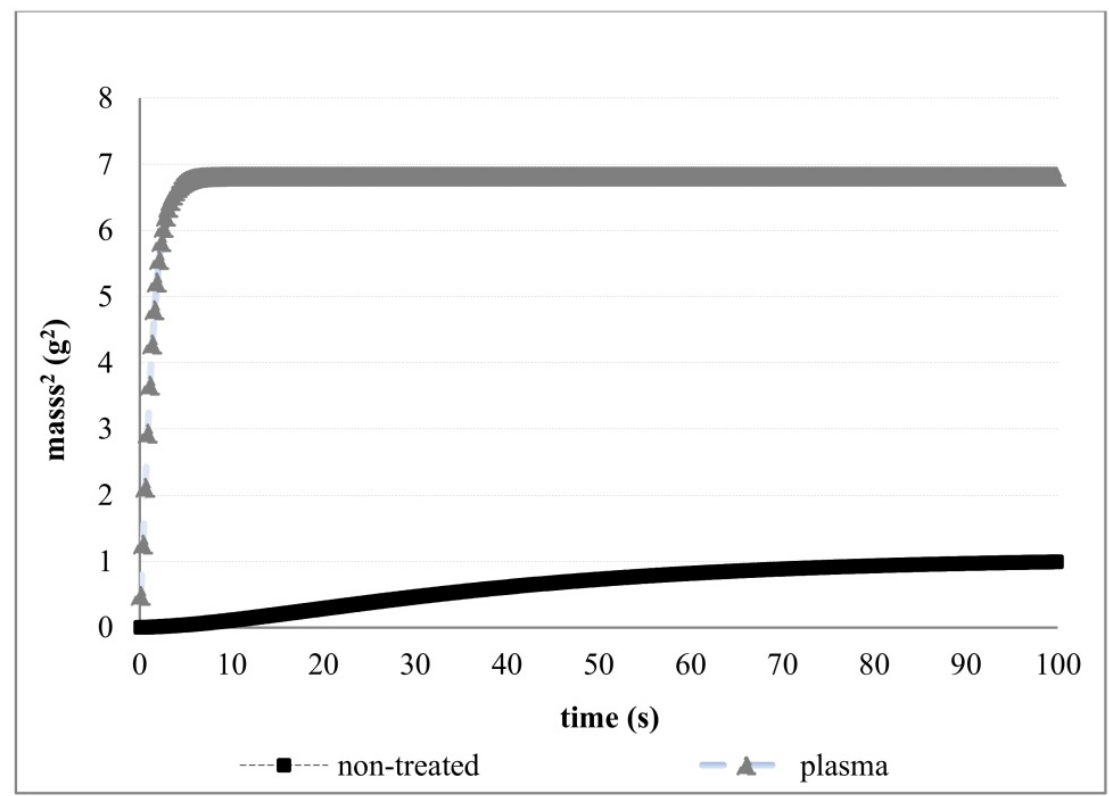

Figure 7. The wetting rise curves for non-treated and oxygen plasma treated cellulose wound dressing obtained with water

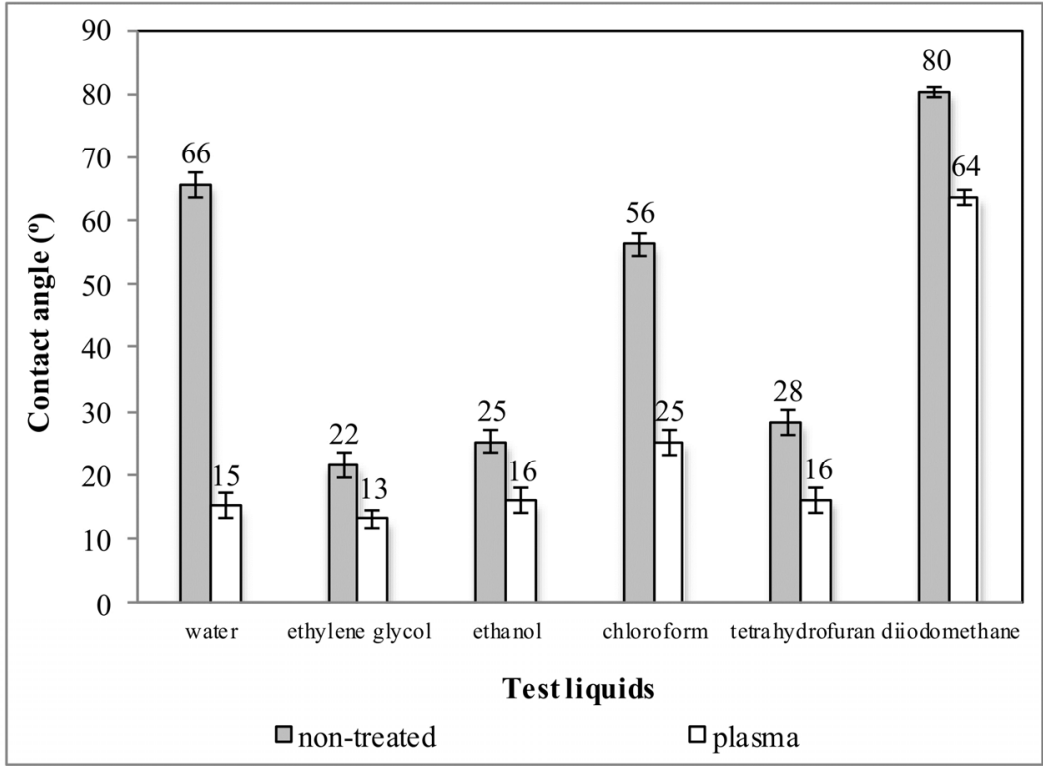

Figure 8. Contact angles of test liquids on non-treated and plasma treated cellulose 
water contact angle determined using water was $66^{\circ}$. As well, similar results were obtained by Hsieh et al., where the water contact angle varied from $56^{\circ}$ to $60^{\circ}$ depending on measuring the contact angle on a single fibre or on a woven fabric [70]. Simoncic et al. obtained even higher values, i.e. $73^{\circ}$ for a plane-wave cotton sample [71], and $80^{\circ}$ for a desized and alkaline scoured cotton samples [72]. In addition, modified Washburn equation as applied procedure for porous samples leads to overestimated contact angle values compared to those measured directly on smooth surfaces of the same solid, if such surface can be obtained at all $[69,73]$. The results indicate a decrease of contact angles for all liquids on plasma treated samples; most effect is evident when using water as the test liquid. Contact angle decrease is explained by increased polarity due to plasma activation.

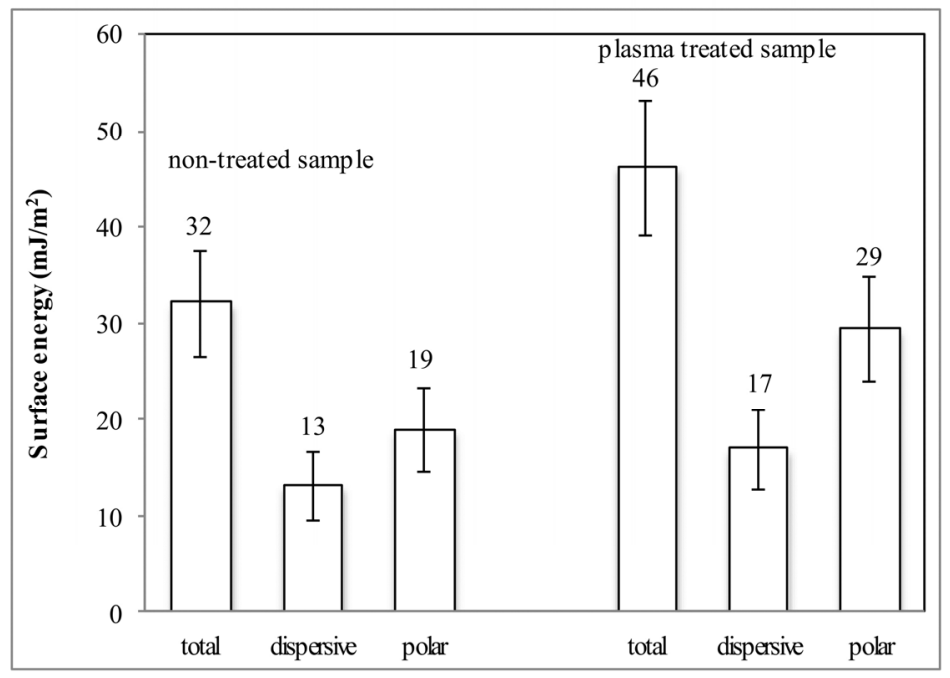

Figure 9. The total surface energy, their dispersive and polar components of non-treated and plasma treated cellulose, as evaluated using OW model

As discussed earlier by other authors [68-69, 74-75], the values of SFEs and their components depend on the choice of solvents and on the combination of the test liquids chosen. In addition, it should be kept in mind that independently of the applied approaches, the obtained values for surface free energy and its components are relative ones and regarded as indicators of the adhesive properties of the surfaces and not as measures of their actual SFEs. Therefore studies on new approaches and experimental procedures to solve the problems are still needed.

Both XPS and SFE results indicate important modifications of the surface properties of cellulose as a consequence of plasma treatment. Formation of oxygen rich functional groups on the cellulose surface causes an increase of the surface energy, especially the polar component. Although the techniques cannot reveal the mechanisms involved in interactions of oxygen plasma with cellulose materials it is possible to explain the results by taking advantage of the known properties of oxygen plasma. Experiments on modification of 
cellulose materials presented in this work have been performed using weekly ionised oxygen plasma. As mentioned earlier, such plasma created in a glass-discharge chamber is rich in neutral reactive particles. The density of charged particles in our plasma is about $10^{15}$ $\mathrm{m}^{3}$. The corresponding flux of ions onto the surface is of the order of $10{ }^{17} \mathrm{~m}^{-2} \mathrm{~s}^{-1}$. This flux is rather low specially when taking into account a very large surface of cellulose materials used in wound dressings. The modification of surface properties thus cannot be explained by interaction of positively charged oxygen ions with solid material. The functionalization of material is rather explained by interaction with particles which abound in our plasma. The non-equilibrium gas is rich in neutral oxygen molecules excited to the first state, and the dissociation fraction is also very high. The excited molecules and neutral oxygen atoms are therefore appropriate candidates for explanation of the observed surface properties. Unfortunately, very little work has been reported on interaction of excited oxygen molecules with solid materials. Many authors agree that probability for surface de-excitation of molecules with the excitation energy of only $1 \mathrm{eV}$ is always low and practically independent from characteristics of solid materials. On the other hand, the literature on interaction between neutral oxygen atoms and solid materials abounds [76-80].

Different authors reported moderate interaction probabilities. The chemical reactivity definitely depends on the type of organic material, but it seems that all authors observed rapid functionalization of practically all organic materials upon exposure to oxygen atoms. The improved surface properties of cellulose material are therefore explained by chemical interaction between neutral oxygen atoms in the ground state and cellulose materials. The interaction leads to formation of oxygen rich functional groups. The etching of cellulose material upon treatment with oxygen atoms also occurs but it can be considered as a minor effect as long as the temperature of cellulose materials remains low. The etching is illustrated in Figure 10, which presents high resolution scanning electron microscope images of non-treated and plasma treated cellulose materials.
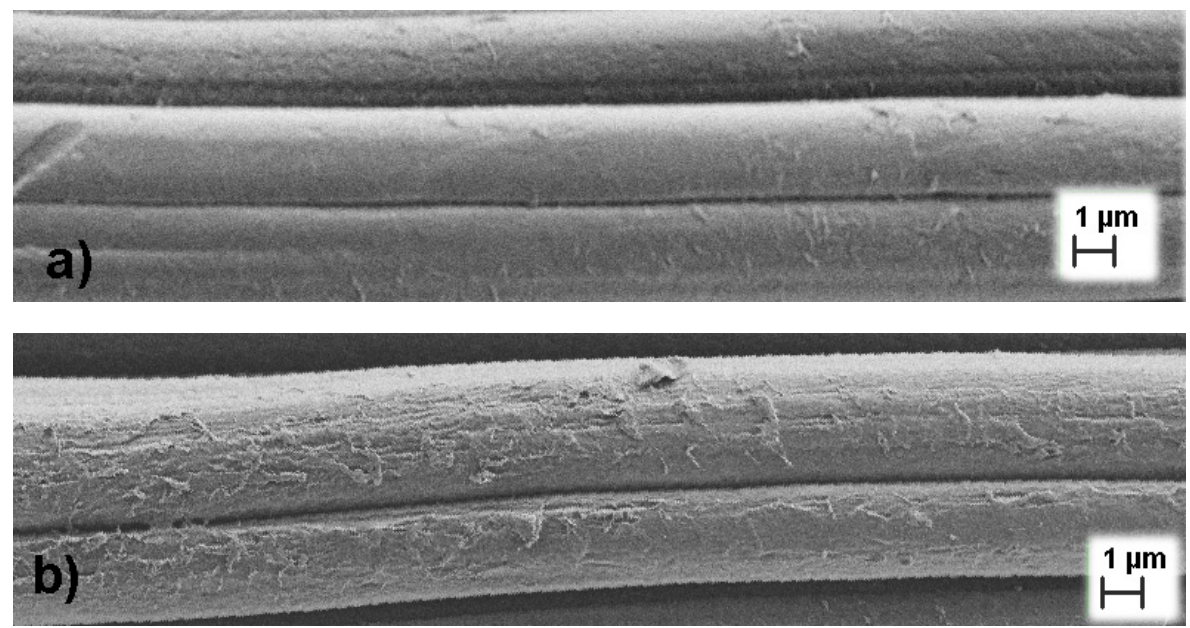

Figure 10. SEM images of non-treated (a) and plasma treated (b) cellulose fibres 
Figure 10 reveals a richer surface morphology of cellulose fibres treated by oxygen plasma. Obviously, some etching occurred during plasma treatment. This observation is sound with effects reported in appropriate literature. Namely, many authors have shown that a treatment of organic materials with gaseous plasma leads to increased surface roughness [77, 81-83]. While the effect is not yet fully understood, a plausible explanation is selective interaction with amorphous and crystalline segments of cellulose materials. Namely, the interaction between neutral oxygen atoms and organic materials is extremely selective. It has to be pointed out that even chemically identical materials are etched at different rates upon exposure to oxygen plasma [84]. In the cited reference [84] major differences in the behaviour of PET polymer with different degrees of crystallinity were reported.

An important consideration associated with plasma treatment is modification of bulk properties of plasma treated materials. A suitable method for estimation of such modifications is measurement of the breaking force and elongation. Figure 11 represents typical results of such measurements. The results are rather surprising: the breaking force does not decrease after plasma treatment but even a small increase is observed, while the elongation is reduced. Since the breaking force and elongation should not depend much on plasma treatment, the results are difficult to explain. Still, the observed improve of the mechanical properties might be explained by increased surface wettability. Namely, the attraction force between particular fibres in the cellulose material may increase as a result of the surface functionalization. Therefore the shrinkage of the origin emptiness between longitudinal and horizontal placed fibres occurred resulting in more compact structure of the material. In any case, the modification of the mechanical properties is not very relevant for the application of cellulose materials in wound dressings since they are designed for regular changing and disposal (and not washing).

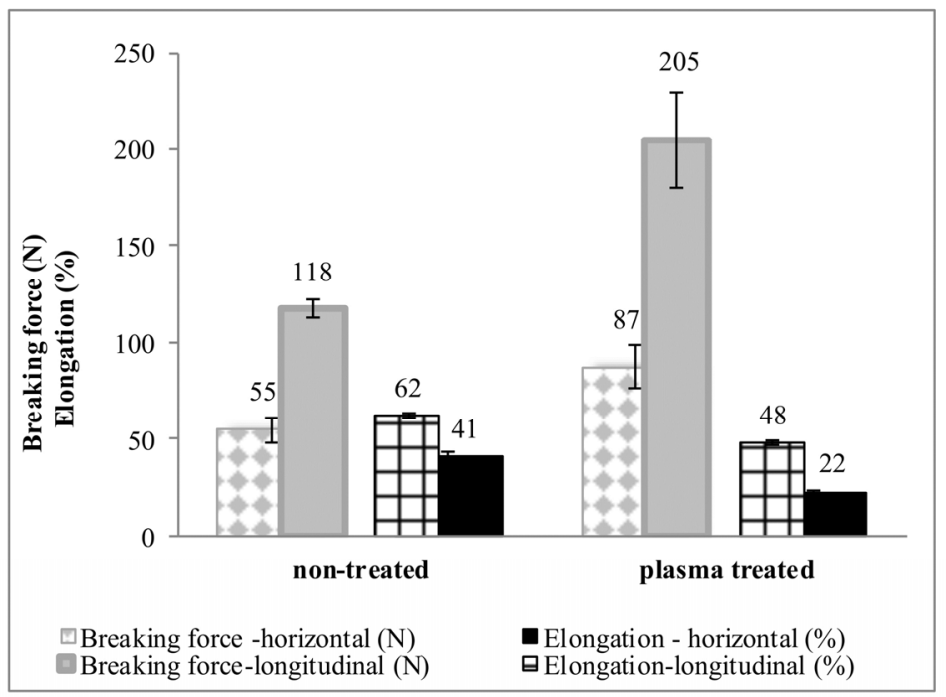

Figure 11. The breaking force and elongation for non-treated and plasma treated cellulose materials 
Finally is worth mentioning that cellulose materials for wound dressings are usually not used directly after surface modifications. An important consideration is ageing of plasma treated materials [77, 85-87]. The surface functional groups are found in non-equilibrium state and any thermodynamically non-equilibrium system tends to approach the equilibrium value. Oxygen rich functional groups may decay by spontaneous release of excessive oxygen, may re-orient from the surface towards the bulk, or may be simply blocked by adsorption of gaseous molecules present in the atmosphere. In order to address the ageing properly, a set of experiments have been performed. Table 4 reveals time dependency of plasma activation effects presented as the loss of oxygen, as determined by XPS and water contact angle results on cellulose measured 1 and 4 days after activation treatment was performed. It is found that the loos rates are within the limits of the experimental error, so it can be concluded that functional groups formed on the surface of cellulose upon exposure to weakly ionized highly dissociated oxygen plasma are rather stable. Figure 12 presents the results of breaking force and elongation of cellulose materials after 10 days since plasma treatment. Again, any modification of these parameters is within the limits of the experimental error. Still, it can be observed that the water contact angle slowly increases with increasing ageing time (see Table 4), but the increase is not dramatic, which indicates that plasma modification of cellulose materials for wound dressings is suitable for practical applications. To demonstrate this, sorption properties evaluated by two different solutions (i.e. synthetic exudate and blood) to simulate real system, were used. As presented in Figure 13, plasma treated samples revealed as effective absorbent for wound fluids i.e. synthetic exudate and blood. The results evident a 3-times faster up-taking of blood and a 15-times faster sorption of exudate by plasma treated samples. One can conclude that the plasma effect was not as significant as by water sorption (see Figure 7), but one should keep in mind that chemical composition of wound fluids are different. Wound exudate may be beneficial, but it can also be problematic, especially in chronic wounds such as leg ulcers. In leg ulcer patients, exudate levels may be high even from small ulcers. Nevertheless, the obtained results illustrate the value of using plasma modified cellulose material as a dressing in order to control exudating by facilitating and supporting the healing of the wound.

\begin{tabular}{|l|l|l|l|}
\hline \multirow{2}{*}{} & \multicolumn{4}{l}{ Time dependency of plasma activation performance } \\
\cline { 2 - 4 } & Immediately after & 1 day after & 4 days after \\
\hline Surface composition & 52.1 & 51.9 & 52.8 \\
\hline $\mathrm{C}($ at. \%) & 47.9 & 48.2 & 47.3 \\
\hline $\mathrm{O}($ at. \%) & 0.91 & 0.92 & 0.89 \\
\hline O/C ratio & \multicolumn{5}{l}{} \\
\hline Hydrophilicity & $17 \pm 1$ & $16 \pm 2$ \\
\hline Water contact angle $\left(^{\circ}\right)$ & $15 \pm 2$ &
\end{tabular}

Table 4. The elemental surface compositions and water contact angles of plasma treated cellulose as a function of ageing 


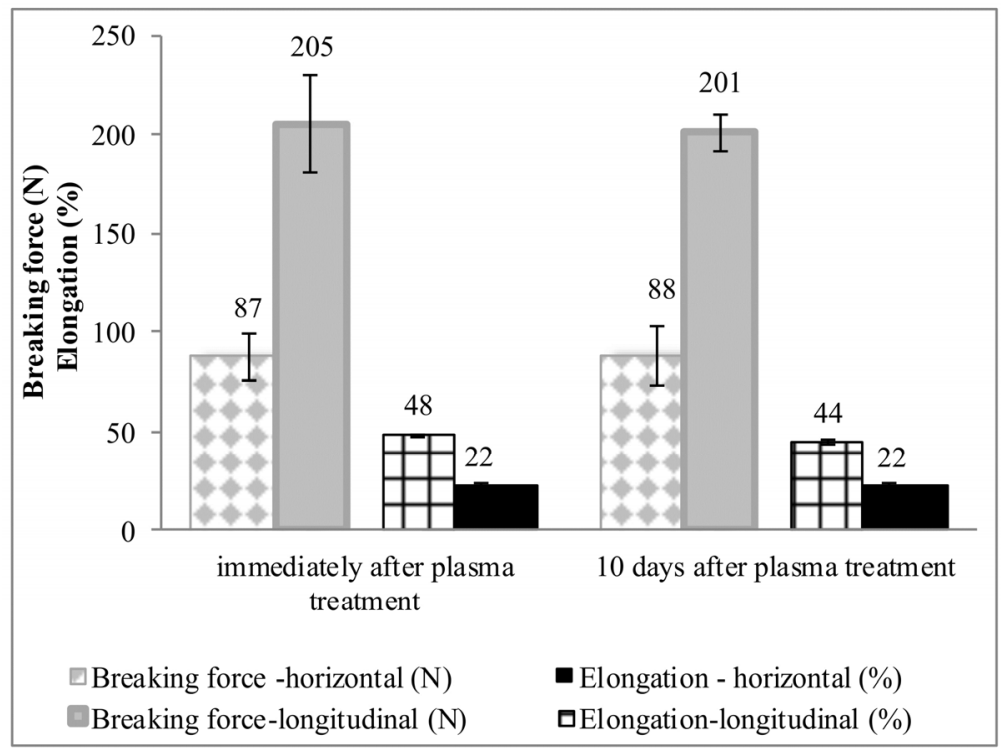

Figure 12. Breaking force and elongation of plasma treated cellulose samples as a function of 10-days ageing

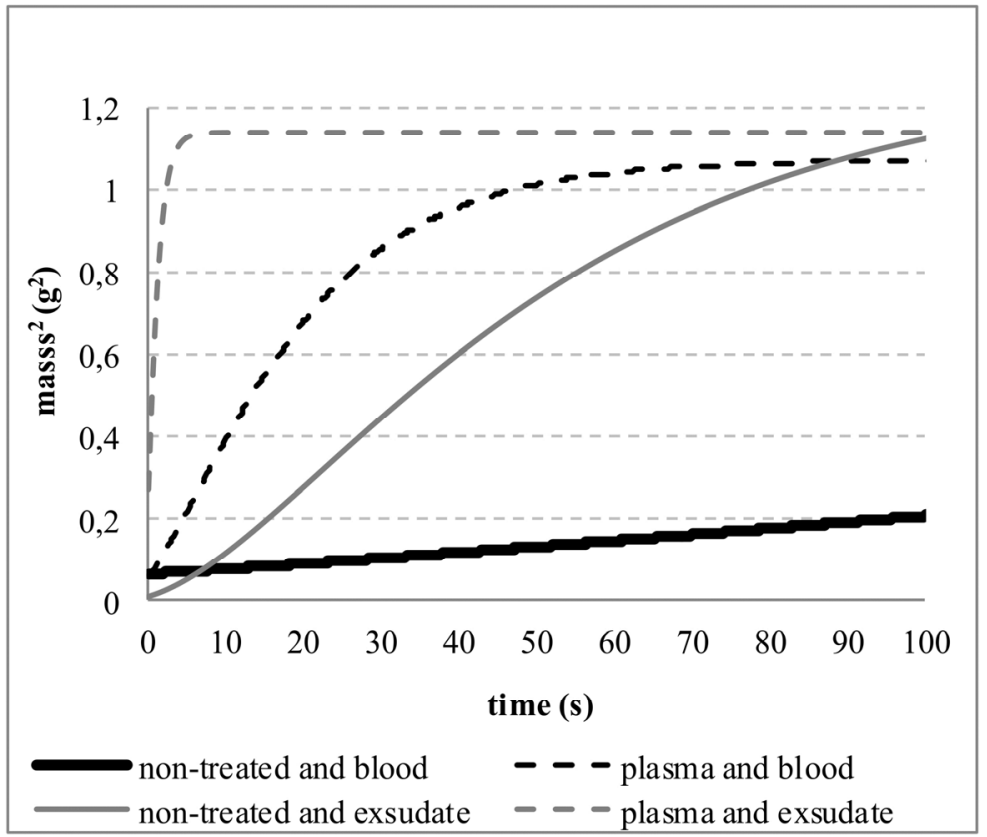

Figure 13. The wetting rise curves for non-treated and oxygen plasma treated cellulose obtained during synthetic exudate and blood sorption 


\section{Conclusion}

Oxygen plasma treatment is a suitable method to improve the quality of cellulose materials. The drastically improvement in sorption capacity far outweigh the minor changes in mechanical properties; while no changes in morphology was observed. Ageing of plasma activation effect was insignificant present within a few days concerning hydrophilicity and tensile properties. Since oxygen plasma contains a variety of different reactive particles, the proper choice of plasma parameters is the key issue. Plasma containing rather large concentration of charged particles is better to be avoided since it usually leads to excessive thermal effects and thus degradation of the material. The available energy dissipated on the cellulose surface at the interaction between charged particles and solid material is simply too large to assure for modification of cellulose material at low temperature. Instead of moderately ionised plasma it is better to use extremely weekly ionised plasma with a large density of neutral reactive particles. The best species in term of reasonable chemical reactivity and low thermal load are neutral oxygen atoms in the ground state. Plasma rich with these particles, but poor in charged particles, is created using an electrodeless high frequency discharge. The absence of electrodes in such discharges is favourable since it prevents substantial drain of neutral oxygen atoms by heterogeneous surface recombination. Best materials for construction of a suitable plasma reactor are different glasses and some ceramics. The plasma facing components should be smooth and should not allow for chemisorption of neutral oxygen atoms. If this requirements are fulfilled the dissociation fraction of oxygen molecules in gaseous plasma at pressures up to several mbar, exceeds the ionisation fraction for at least five orders of magnitudes. Such plasma is therefore safe to use since it does not lead to excessive exothermic reactions and thus thermal degradation of treated materials. Based on that, plasma treatment is advantage surface activation procedure in order to obtain super-hydrophilic matric, used as a potential layer in wound dressings.

\section{Author details}

Zdenka Persin, Tina Maver and Karin Stana Kleinschek*

Laboratory for Characterisation and Processing of Polymers, Faculty of Mechanical Engineering, University of Maribor, Maribor, Slovenia

Zdenka Persin, Alenka Vesel, Tina Maver, Uros Maver and Karin Stana Kleinschek Centre of Excellence for Polymer Materials and Technologies, Ljubljana, Slovenia

Alenka Vesel

Jozef Stefan International Postgraduate School, Ljubljana, Slovenia

Miran Mozetic

Jozef Stefan Institute, Ljubljana, Slovenia

\footnotetext{
${ }^{*}$ Corresponding Author
} 


\section{Acknowledgement}

This work was supported by the Ministry of Higher Education, Science and Technology of the Republic of Slovenia [Grant number 3211-10-000057].

\section{References}

[1] Freytag R., Donzé J.J (1983) Alkali treatment of cellulose fibres. In Lewin M, Sello SB, editors. Handbook of fiber science and technology: Volume I. Chemical processing of Fibers and Fabrics, Fundamentals and Preparation, Part A. New York and Basel: Marcel Deckker, Inc. pp. 91-121.

[2] Lewin M. (1984) Bleaching of Cellulosic and Synthetic Fabrics. In Lewin M, Sello SB, editors. Handbook of fiber science and technology: volume I. Chemical processing of Fibers and Fabrics, Fundamentals and Preparation, Part B. New York and Basel: Marcel Deckker, Inc. pp. 91-243.

[3] Durso D.F (1978) Chemical modification of cellulose - A historical review. In Rowell RM, Young RA, editors. Modified Cellulosics. NY, ZDA: Academic Press. pp. 23-37.

[4] Fengel D., Wegener G. (1984) Reactions in alkaline medium. In: Fengel D, Wegener G, editors. Wood, Chemistry, Ultrastructure, Reactions. Berlin, New York: Walter de Gruyter. pp. 66-105.

[5] Gorjanc M, Bukosek V, Gorensek M, Vesel A (2010) The influence of water vapor plasma treatment on specific properties of bleached and mercerized cotton fabric. Tex. Res. J. 80: 557-567.

[6] Vesel A, Mozetic M, Strnad S, Stana-Kleinschek K, Hauptman N, Persin Z (2010) Plasma modification of viscose textile. Vacuum 84: 79-82.

[7] Gorensek M, Gorjanc M, Bukosek V, Kovac J, Petrovic Z Lj, Puac N (2010) Functionalization of polyester fabric by Ar/N2 plasma and silver. Tex. Res. J. 80:16331642.

[8] Radetic M, Jovancic P, Puac N, Petrovic Z Lj (2005) Environmental impact of plasma application to textiles. J. Phys.: Conf. Series, 71: 12017 doi:10.1088/17426596/71/1/012017. Available: http://iopscience.iop.org/1742-6596/71/1/012017. Accessed 2012 April $6^{\text {th }}$.

[9] Mihailovic D, Saponjic Z, Molina R, Puac N, Jovancic P, Nedeljkovic J, Radetic M (2010) Improved properties of oxygen and argon RF plasma-activated polyester fabrics loaded with $\mathrm{TiO}_{2}$ nanoparticles. ACS applied materials and interfaces. 2 (6): 1700-1706.

[10] Canal C, Gaboriau F, Villeger S, Cvelbar U, Ricard A (2009) Studies on antibacterial dressings obtained by fluorinated post-discharge plasma. Int. J. Pharm. 367 (1-2): 155161.

[11] Canal C, Villeger S, Cousty S, Rouffet B, Sarrette JP, Erra P, Ricard A (2008) Atomsensitive textiles as visual indicators for plasma post-discharges. Appl. Surf. Sci. 254 (18): 5959-5966.

[12] Ristic N, Jovancic P, Canal C, Jocic D (2009) One-bath one-dye class dyeing of PES/cotton blends after corona and chitosan treatment. Fiber Polym. 10 (4): 466-475. 
[13] Gorensek M, Gorjanc M, Bukosek V, Kovac J, Jovancic P, Mihailovic D (2010) Functionalization of PET fabrics by corona and nano silver. Tex. Res. J. 80/3: 253-262.

[14] Guimond S, Hanselman B, Amberg M, Hegemann D (2010) Plasma functionalization of textiles: Specifics and possibilities. Pure Appl. Chem. 82 (6): 1239-1245.

[15] Morent R, De Geyter N, Verschuren J, De Clerck K, Kiekens P, Leys C (2008) Nonthermal plasma treatment of textiles. Surf. Coat. Technol. 202: 3427-3449.

[16] Canal C, Erra P, Molina R, Bertran E (2007) Regulation of surface hydrophilicity of plasma treated wool fabrics. Tex. Res. J. 77 (8): 559-564.

[17] Devetak M, Skoporc N, Rigler M, Persin Z, Drevensek-Olenik I, Copic M, StanaKleinschek K (2012) Effects of plasma treatment on water sorption in viscose fibres. Mater. Tehnol. 46 /1 69-73.

[18] Ferrero F (2003) Wettability measurements on plasma treated synthetic fabrics by capillary rise method. Polym. Test. 22 (5): 571-578.

[19] Persin Z, Stenius P, Stana-Kleinschek K (2011) Estimation of the Surface Energy of Chemically and Oxygen Plasma-Treated Regenerated Cellulosic Fabrics Using Various Calculation Models. Tex Res J. 81/16: 1673-1685.

[20] Persin Z, Stana-Kleinschek K, Sfiligoj-Smole M, Kreze T, Ribitsch V (2004) Determining the Surface Free Energy of Cellulose Materials with the Powder Contact Angle Method. Tex Res J. 74/1: 55-62.

[21] Hua ZQ, Sitaru R, Denes F, Young RA (1997) Mechanisms of oxygen- and argon-RFplasma-induced surface chemistry of cellulose. Plasmas Polym. 2: 199-224.

[22] Alfredo Calvimontes, Peter Mauersberger, Mirko Nitschke, Victoria Dutschk and Frank Simon, Effects of oxygen plasma on cellulose surface, Cellulose, 18 (2011) 803-809.

[23] Inbakumar S, Morent R, De Geyter N, Desmet T, Anukaliani A, Dubruel P, Leys C (2010) Chemical and physical analysis of cotton fabrics plasma-treated with a low pressure DC glow discharge. Cellulose 17: 417-426.

[24] Vander Wielen LC, Ragauskas AJ (2004) Grafting of acrylamide onto cellulosic fibers via dielectric-barrier discharge. Eur. Polym. J. 40/3: 477-482.

[25] Jun W, Fengcai Z, Bingqiang C (2008) The Solubility of Natural Cellulose After DBD Plasma Treatment. Plasma Sci. Technol. 10/743. Available:

http://iopscience.iop.org/1009-0630/10/6/18 (doi:10.1088/1009-0630/10/6/18). Accessed: 2012 April $6^{\text {th }}$.

[26] Ercegovic Razic S, Cunko R, Svetlicic V, Segota S (2011) Application of AFM for identification of fibre surface changes after plasma treatments. Mater. Technol.: Adv. Perform. Mater. 26: 146-152.

[27] Mahlberg R, Niemi HEM, Denes FS, Rowell RM (1999) Application of AFM on the Adhesion Studies of Oxygen-Plasma-Treated Polypropylene and Lignocellulosics. Langmuir. 15: 2985-2992.

[28] Ward TL, Jung HZ, Hinojosa O, Benerito RR (1978) Effect of rf cold plasmas on polysaccharides. Surf. Sci. 76: 257-273.

[29] Wong KK, Tao XM, Yuen CWM, Yeung KW (1999) Low Temperature Plasma Treatment of Linen. Tex. Res. J. 69: 846-855.

[30] Strnad J (1977) Fizika 1. Ljubljana: DZS. 256 p. 
[31] Ricard A (1996) Reactive Plasmas, 1 edn. Paris: SFV.

[32] Kutasi K, Guerra V, Sá PA (2011) Active species downstream of an Ar-O2 surface-wave microwave discharge for biomedicine, surface treatment and nanostructuring. Plasma Sources Sci. Technol. 20/3: doi:10.1088/0963-0252/20/3/035006. Available: http://iopscience.iop.org/0963-0252/20/3/035006/. Accessed: 2012 April $6^{\text {th }}$.

[33] Mozetic M, Cvelbar U, Vesel A, Ricard A, Babic D, Poberaj I (2005) A diagnostic method for real-time measurements of the density of nitrogen atoms in the postglow of an ArN2 discharge using a catalytic probe. J. Appl. Phys. 97/ 10: 103308-1-103308-7. Available: http://dx.doi.org/10.1063/1.1906290. Accessed: 2012 April $6^{\text {th }}$.

[34] Mozetic M, Vesel A, Cvelbar U, Ricard A (2006) An iron Catalytic Probe for Determination of the O-atom Density in an Ar/O2 Afterglow. Plasma Chem. Plasma P. 26/ 2: 103-117.

[35] Zaplotnik R, Vesel A, Mozetic M (2012) Fiber Optic Catalytic Sensor for Neutral Atom Measurements in Oxygen Plasma. Sensors. 12: 3857-3867.

[36] Drenik A, Cvelbar U, Ostrikov K, Mozetic M (2008) Catalytic probes with nanostructured surface for gas/discharge diagnostic: a study of a probe signal behaviour. J. Phys. D Appl. Phys. 41/11: 115201-1-115201-7.

[37] Balat-Pichelin M, Vesel A (2006) Neutral oxygen atom density in the Mesox air plasma solar furnace facility. Chem. Phys. 327/1: 112-118.

[38] Vesel A, Mozetic M, Balat-Pichelin M (2007) Oxygen atom density in microwave oxygen plasma. Vacuum. 81/9: 1088-1093.

[39] Primc G, Zaplotnik R, Vesel A, Mozetic M (2011) Microwave discharge as a remote source of neutral oxygen atoms. AIP Advances. 1/2: 022129-1-022129-11. Available: http://dx.doi.org/10.1063/1.3598415. Accessed 2012 April 6th.

[40] Denysenko I, Ostrikov K, Azarenkov NA (2009) Dust charge and ion drag forces in a high-voltage, capacitive radio frequency sheath. Phys. Plasmas 16/11: 113707-1 - 113707-10.

[41] Kratzer M, Brinkmann RP, Sabisch W, Schmidt H (2001) Hybrid model for the calculation of ion distribution functions behind a direct current or radio frequency driven plasma boundary sheath. J. Appl. Phys. 90/5: 2169-2179.

[42] Schuengel E, Zhang QZ, Iwashita S, Schulze J, Hou LJ, Wang YN, Czarnetzki U (2011) Control of plasma properties in capacitively coupled oxygen discharges via the electrical asymmetry effect. J. Phys. D Appl. Phys. 44/28: 285205-1 - 285205-14.

[43] Zaplotnik R, Vesel A, Mozetic M (2011) Transition from E to H modes in inductively coupled oxygen plasma: behaviour of oxygen atom density. Europhys. Lett. 95: 55001-1 $-55001-5$.

[44] Takeda K, Takashima S, Ito M, Hori M (2008) Absolute density and temperature of $\mathrm{O}\left({ }^{1} \mathrm{D}_{2}\right)$ in highly Ar or $\mathrm{Kr}$ diluted $\mathrm{O}_{2}$ plasma. Appl. Phys. Lett. 93/2: 021501-1 - 021501-3.

[45] Mozetic M (2012) Application of X-ray photoelectron spectroscopy for characterization of PET biopolymer. Mater. Tehnol. 46/1: 47-51.

[46] Morent R, De Geyter N, Leys C, Vansteenkiste E, De Bock J, Philips W (2006) Measuring the wicking behavior of textiles by the combination of a horizontal wicking experiment and image processing. Rev. Sci. Instrum. 77. Available:

http://dx.doi.org/10.1063/1.2349297. Accessed 2012 March 3rd. 
[47] Grundke K, Bogumil T, Gietzelt T, Jacobasch HJ, Kwok DY, Neumann AW (1996) Wetting measurements on smooth, rough and porous solid surfaces. Prog. Colloid Polym. Sci. 101:58-68.

[48] Chibowski E, Perea-Carpio R (2001) A Novel Method for Surface Free-Energy Determination of Powdered Solids. J Colloid Interf Sci. 240/2: 473-479.

[49] Dang-Vu T, Hupka J (2005) Characterization Of Porous Materials By Capillary Rise Method. Physicochem Probl Mi. 39: 47-65.

[50] Carroll B.J (1993) Direct measurement of the contact angle on plates and on thin fibres: some theoretical aspects. In: Mitall KL, editor. Contact angle, wetting, and adhesion. The Netherlands: VSP BV. pp. 235-246.

[51] Chan C.M (1994) Contact angle measurements. In Chan CM, editor. Polymer surface modification and characterization. Münich Vienna New York: Carl Hanser Verlag. pp. 35-76.

[52] Good R.J (1993) Contact angle, wetting, and adhesion: a critical review. In: Mitall KL, editor. Contact angle, wetting, and adhesion. The Netherlands: VSP BV.pp.3-37.

[53] Fowkes FM (1962) Determination of interfacial tensions, contact angles, and dispersion forces in surfaces by assuming additivity of intermolecular interactions in surfaces. J Phys Chem. 66: 382-382.

[54] Fowkes FM (1964) Attractive forces at interfaces. Ind Eng Chem. 56: 40-52.

[55] Zisman W.A (1964) Relation of the equilibrium contact angle to liquid and solid constitution. In: Fowkes FM editor. Contact angle, wettability and adhesion. Washington, DC: American Chemical Society. pp. 1-51.

[56] Kwok DY, Neumann AW (1999) Contact angle measurement and contact angle interpretation. Adv Colloid Interface Sci. 81: 167-249.

[57] Rabel W (1977) Flüssigkeitsgrenzflächen in Theorie und Anwendungstechnik. Physkalische Blätter. 33: 151-161.

[58] Kaelble DH (1969) Peel adhesion: Influence of surface energies and adhesive rheology. J Adhes. 1: 102-123.

[59] Kaelble DH (1972) Physical chemistry of adhesion. New York: Wiley Interscience. Chapter 9.

[60] Owens DK, Wendt RC (1969) Estimation of the surface free energy of polymers. J Appl Polym Sci. 13: 1741-1747.

[61] van Oss CJ, Chaudhury MK, Good RJ (1988) Additive and nonadditive surface tension components and the interpretation of contact angles. Langmuir. 4: 884-891.

[62] van Oss CJ (1993) Acid-base interfacial interactions in aqueous media. Colloids Surf. A. 78: $1-49$.

[63] van Oss CJ, Chaudhury MK, Good RJ (1986) The role of van der Waals forces and hydrogen bonds in "hydrophobic interactions" between biopolymers and low energy surfaces. J Colloid Interface Sci. 111: 378-390.

[64] Good RJ, Girifalco LA (1960) A theory for estimation of interfacial energies III. Estimation of surface energies of solids from contact angle data. J Phys Chem. 64: 561565. 
[65] Jakobasch H.J, Grundke K, Mäder E, Freitag K.H, Panzer U (2003) Application of the surface free energy concept in polymer processing. In: Mittal KL editor. Contact angle, wettability \& adhesion. The Netherlands: Zeist, VSP BV. pp. 921-936.

[66] Washburn EW (1921) The dynamics of capillary flow. Phys Rev. 17/3: 273-283.

[67] Grundke K, Boerner M, Jacobasch HJ (1991) Characterization of fillers and fibres by wetting and electrokinetic measurements. Colloid Surface. 58/1-2: 47-59.

[68] Fowkes FM, Riddle FL, Pastore WE, Weber AA (1990) Interfacial interactions between self-associated polar liquids and squalane used to test equations for solid-liquid interfacial interactions. Colloids Surf. 43: 367-387.

[69] Chibowski E, Perea-Carpio R (2002) Problems of contact angle and solid surface free energy determination. Adv Colloid Interface Sci. 98: 245-264.

[70] Hsieh YL, Yu B (1999) Liquid Wetting, Transport, And Retention Properties Of Fibrous Assemblies, Part I: Water Wetting Properties Of Woven Fabrics And Their Constituent Single Fibres. Textile Res. J. 62 (11): 677-685.

[71] Simončič B, Černe L, Tomšič B, Orel B(2008) Surface properties of cellulose modified by imidazolidinone. Cellulose 15:47-58.

[72] Simončič B, Rozman V (2007) Wettability of cotton fabric by aqueous solutions of surfactants with different structures. Colloids and Surfaces A: Physicochem. Eng. Aspects 292 236-245.

[73] Chibowski E (2000) Thin layer wicking - Methods for the determination of acid-base free energies of interaction,. In Mittal KL, editor. Acid-base Interactions: Relevance of Adhesion, Science and technology, Vol.2. Utrech, The Nethelands, VSP BV 2000, pp. 419-437.

[74] Della Volpe C, Siboni S (2001) The evaluation of electrondonor and electron-acceptor properties and their role in the interaction of solid surfaces with water. In: Morra M editor. Water in biomaterials surface science. Chichester: John Wiley \& Sons, Ltd. pp. 83-214.

[75] Della Volpe C, Maniglio D, Brugnara M, Siboni S, Morra M (2004) The solid surface free energy calculation I. In defense of the multicoponent approach. J. Colloid Interface Sci. 271: 434-453.

[76] Vesel A (2010) Surf. Modification of polystyrene with a highly reactive cold oxygen plasma. Coat. Technol. 205/2: 490-497.

[77] Vesel A, Junkar I, Cvelbar U, Kovac J, Mozetic M (2008) Surfacemodification of polyester by oxygen-and nitrogen-plasma treatment. Surf. Interface Anal. 40/11: 14441453.

[78] Borcia G, Anderson CA, Brown NMD (2003) Dielectric barrier discharge for surface treatment: Application to selected polymers in film and fibre form. Plasma Sources Sci T. 12/3: 335-344.

[79] Borcia G, Anderson CA, Brown NMD (2004) The surface oxidation of selected polymers using an atmospheric pressure air dielectric barrier discharge. Part II. Appl. Surf. Sci. 225: 186-197.

[80] Chan CM, Ko TM, Hiraoka H (1996) Polymer surface modification by plasmas and photons. Surf. Sci. Rep. 24/1-2: 1-54. 
[81] Walthera F, Heckla WM, Stark RW (2008) Evaluation of nanoscale roughness measurements on a plasma treated SU-8 polymer surface by atomic force microscopy. Appl. Surf. Sci. 254/22: 7290-7295.

[82] Vourdas N, Kontziampasis D, Kokkoris G, Constantoudis V, Goodyear A, Tserepi A, Cooke M, Gogolides E (2010) Plasma directed assembly and organization: bottom-up nanopatterning using top-down technolog. Nanotechnology. 21: 085302-1 - 085302-8.

[83] Vourdas N, Tserepi A, Gogolides E (2007) Nanotextured super-hydrophobic transparent poly(methyl methacrylate) surfaces using high-density plasma processing. Nanotechnology. 18:125304-1 - 125304-7.

[84] Junkar I, Cvelbar U, Vesel A, Hauptman N, Mozetic M (2009) The Role of Crystallinity on Polymer Interaction with Oxygen Plasma. Plasma Processes Polym. 6/10: 667-675.

[85] Larrieu J, Held B, Martinez H, Tison Y (2005) Ageing of atactic and isotactic polystyrene thin films treated by oxygen DC pulsed plasma. Surf. Coat. Technol. 200: 2310- 2316.

[86] Morent R, De Geyter N, Leys C, Gengembre L, Payen E (2007) Study of the ageing behaviour of polymer films treated with a dielectric barrier discharge in air, helium and argon at medium pressure. Surf. Coat. Technol. 201: 7847- 7854.

[87] Yun YI, Kim KS, Uhm SJ, Khatua BB, Cho K, Kim JK, Park CE (2004) Aging behavior of oxygen plasma-treated polypropylene with different crystallinities. J. Adhesion Sci. Technol. 18/11: 1279-1291. 